\title{
Modelling conduction delays in the corpus callosum using MRI-measured g-ratio
}

\author{
Berman S. ${ }^{1}$, Filo S., Mezer A. A. \\ 1. Edmond \& Lily Safra Center for Brain Sciences, The Hebrew University of \\ Jerusalem, Jerusalem, Israel
}

\begin{abstract}
Conduction of action potentials along myelinated axons is affected by their structural features, such as the axonal g-ratio, the ratio between the inner and outer diameters of the myelin sheath surrounding the axon. The effect of $g$-ratio variance on conduction properties has been quantitatively evaluated using single-axon models. It has recently become possible to estimate a g-ratio weighted measurement in vivo using quantitative MRI. Nevertheless, it is still unclear whether the variance in the g-ratio in the healthy human brain leads to significant differences in conduction velocity. In this work we tested whether the g-ratio MRI measurement can be used to predict conduction delays in the corpus callosum.
\end{abstract}

We present a novel framework in which the structural properties of fibers (i.e. length and g-ratio, measured using MRI), are incorporated in a biophysical model of axon conduction, to predict conduction delays of long-range white matter fibers. We applied this framework to the corpus callosum, and found conduction delay estimates that are compatible with previously estimated values of conduction delays. We account for the variance in the velocity given the axon diameter distribution in the splenium, mid-body and genu, to further compare the fibers within the corpus callosum.

Conduction delays have been suggested to increase with age. Therefore, we investigated whether there are differences in the g-ratio and the fiber length between young and old adults, and whether this leads to a difference in conduction speed and delays. We found small but significant differences between the predicted delays of the two groups in the motor fibers of the corpus callosum. We also found that the motor fibers of the corpus callosum have the fastest conduction estimates. Using the axon diameter distributions, we found that the occipital fibers have the slowest estimations, while the frontal and motor fiber tracts have similar estimates.

Our study provides a framework for predicting conduction latencies in vivo. The framework could have major implications for future studies of white matter diseases and large range network computations. Our results highlight the need for improving additional in vivo measurements of white matter microstructure. 


\section{Introduction}

White matter tissue consists of myelinated and non-myelinated axons and glial cells, forming large-scale networks which are necessary for learning (1-3), cognitive functions (4), normal development, and normal aging $(5,6)$. Furthermore, damage to the structure of the white matter has been associated with a variety of diseases and disorders, including multiple sclerosis and schizophrenia $(7,8)$. These networks of white matter fibers are essential for brain function because their main role is to conduct action potentials between distant brain regions. The conduction delays, defines as the time required for information to travel along white matter fibers, could have computational implications. The conduction delays were also shown to vary with many factors, including age (9), sex (10), and white matter diseases such as multiple sclerosis (11).

In vivo anatomical studies of white matter often use indirect measures of its structure and integrity properties, which would benefit from a biophysical framework to relate them to white matter function (12-14). Ex vivo and in vitro studies have led to a rich understanding of the biophysical connection between the axon microstructure and its conduction properties (e.g., (15-17)). Moreover, computational models of conduction of action potentials along myelinated axons provide a way to explore the dependence of the conduction properties on the axons' geometrical and electrical properties.

One such geometrical property of axons is the g-ratio $(g)$. It is defined as the ratio between the inner and outer diameters of the myelin sheath surrounding the axon. For a fixed inner diameter (axon diameter, $d$ ), changing $g$ means changing the myelin sheath thickness, thus affecting membrane resistance and capacitance. For a fixed outer diameter (fiber diameter, $D$ ), changing $g$ means varying both myelin sheath thickness and intra-axonal space, thus affecting axial conductivity as well. Under these assumptions Rushton found analytically that in the peripheral nervous system, an optimal $g$ for conduction velocity is around 0.6 (18). Further work, modelling the central nervous system of the rat, has shown that higher values $(\sim 0.77)$ would be optimal given other constraints on the tissue such as energy consumption or volume limitations (19).

Empirical values of g-ratio can be measured directly using invasive techniques such as electron microscopy. Such studies reveal the $g$ is indeed distributed around near optimal values: the reported values are around 0.67 for monkey's corpus callosum $(20,21), 0.81$ for guinea pigs' optic nerve (22), 0.76 for the mouse corpus callosum (23) and 0.75-0.8 for the rat corpus callosum (24). It has recently become possible to estimate a $g$-weighted measurement in humans in vivo using quantitative MRI (qMRI) (25-29). These measurements provide values and trends that are similar to those found with histology $(21,27)$. Nevertheless, it is unclear whether the variance captured by the in vivo $g$ measurements in the healthy human brain can be translated to meaningful differences in conduction velocity.

Theoretical estimates of conduction time across the corpus callosum have been previously used in cross-species studies (30). In vivo estimations of callosal delays can be done 
41 using electrical recordings such as EEG or MEG. Such measurements have been shown to be related to callosal structure $(31,32)$, and are likely to increase with age $(33,34)$. The increase in callosal delay time with age could be related to white matter structure, as it has been shown that during aging the brain tissue structure undergoes changes, with accumulating damage to the white matter $(35,36)$, and a decrease in myelination (37). It

46 is still an open question whether $g$-weighted MRI can be related to age dependent 47 changes in callosal delay time.

48 Both theoretical work, and in vivo studies (mentioned above) show a relationship between indirect measurements of white matte structure and function, yet it is important to establish this relationship empirically. Animal studies that provide estimates of both axon geometry and conduction velocity are rare, particularly in mammalian central nervous system. Nevertheless, there is some empirical evidence for the relationship between axon diameter, myelin thickness and conduction velocity. Hursh used excised nerves of the cat to display a linear relationship between axon diameter and conduction velocity (38). A similar dependence was found on the frog sciatic nerve (39). Furthermore, a strong relationship was found between myelin thickness and conduction velocity in the rabbit peroneal nerve (40).

The results of the animal studies are in agreement with the theoretical work, and both have existed for dozens of years, highlighting a great need for understanding the functional meaning of structural properties of white matter. Recent advances in qMRI measurements allow to estimate white matter microstructure in vivo. Importantly, it is still unclear how the structural measurements done with MRI can be biophysically related to conduction along the white matter fibers. To test whether the $g$-weighted MRI measurements can be used to model callosal delays, we first discuss a theoretical framework that can be used to calculate conduction velocity for a single axon. Next, we describe how we implement this model to calculate conduction delays in the corpus callosum in vivo with MRI. This is the first time a framework is presented that related MRI measurements in white matter to conduction properties using biophysical models of conduction in myelinated axons. Finally, we use our framework to estimate conduction in both younger (under 40) and older (over 65) subjects. Thus, we test whether incorporating the $g$-weighted MRI measurement in a biophysical model of conduction allows us to explain the previously observed increase

72 in delay as function of age.

\section{2. Theory - predicting conduction latencies}

74 In the theory section we propose a framework that relates MRI measurements of averages of microstructure features to single axon conduction models, in order to predict white matter conduction in vivo. For this purpose, we first identify a relevant axon model, and the essential parameters in the model (section 2.1). Then, we describe the available qMRI parameters that can be used to estimate those model parameters (section 2.2). For model parameters that cannot be estimated based on qMRI measurements, specific values must be assumed. We will describe the assumptions underlying the choice of specific values 
81 for those parameters (section 2.3). In this section we also discuss how these parameters can be applied to a model for predicting conduction along a certain tract.

The myelinated fiber is comprised of two qualitatively different sections: the nodes of Ranvier and the internodes. The nodes of Ranvier, which contain a high density of voltagegated $\mathrm{Na}+$ channels can generate action potentials. The internodes are long sections between the nodes (about $10^{3}$ longer), and they are coated by many layers of myelin sheaths (41). Due to the myelin sheath, the voltage progression in the internode can be described as passive. The progression of voltage along both segments of the axons have already been modelled and numerically simulated (e.g., 12,24-26). This understanding of the axon signal conduction, together with knowledge on the axon's electrical properties $(45,46)$ and the ability to measure its geometry, will allow to implement a model that links white matter structure with function in biophysical terms.

\subsubsection{Dependence of conduction velocity on axon geometry}

The determinants of conduction velocity of action potential propagation along a myelinated axon have been previously described and modelled. Rushton (18) was the first to describe the dependence of the conduction velocity of a myelinated axon on its geometry. Rushton showed that for a myelinated axon, the conduction velocity $(\theta)$ is proportional to internode length $(l)$. The internode length, in turn, is roughly proportional to the fiber diameter $(D)$, which can be defined using the axon diameter $(d)$ and the g-ratio $(g): D=d / g$. According to his formulation, we could predict conduction velocity, up to a proportionality factor, using the axon diameter and $g$ (equation 1): $\theta \propto l \propto D g \sqrt{-\ln (g)} \propto d \sqrt{-\ln (g)}$ Rushton's formulation provides an expression proportional to conduction velocity. Following studies have replicated Rushton's predictions and shown the dependence of the axon's conduction velocity on its diameter, internode length, and myelin sheath thickness (47-50). Others studies evaluated the effect of additional parameters of the axon on conduction velocity. For example, Moore et al. (42), using numerical simulations of myelinated fibers, examined both geometrical and physiological parameters. The simulations replicated the results showing a dependence of conduction velocity on internode properties, while also revealing that node parameters have a minimal effect on the conduction velocity.

112 Moore's approach of numerically simulating the voltage propagation, provides an 113 approximation of conduction velocity, and allows a 'direct' manipulation of the different 114 axon properties, which could be useful for the purpose of this study. In this study we use a recent MATLAB implementation that numerically simulates the conduction along a myelinated axon using Richardson's axon model-C, and Halter and Clark's numerical formalization $(43,51,52)$. Specifically, the simulation models the axon by dividing it into compartments representing the node, paranode and internode. For each time step, current 
121 rate, and the specific membrane capacitance and conductance. The intracellular axial 122 current flow is calculated from the specific intracellular resistance and the gradient of 123 intracellular voltage. In the simulations, each model axon was subjected to a current 124 stimulation of amplitude $3 \mathrm{nA}$ applied for $10 \mathrm{~ms}$ to the first node. The conduction velocity 125 was then measured over a 10-node interval between the 20th and 30th node. The 126 simulations were checked to ensure membrane potential peak of at least $-20 \mathrm{mV}$ were 127 achieved on a minimum of 10 consecutive nodes.

\section{2.2. The measurements in $q M R I$}

129 Using a biophysical model, such as the simulation described here, allows for the 130 computation of the conduction time of an action potential along a "theoretical axon", which 131 represents an average axon in an axonal fascicle. In order to do so in vivo, we should be 132 able to measure white matter pathways and estimate their microstructure. In particular, in 133 the section below we describe ways to measure the necessary geometrical 134 measurements: the axon diameter, $g$-ratio, and axon length. In the following subsections, 135 we describe recent advances in qMRI, which allow for ongoing improvement in estimating 136 the axon diameter, the g-ratio and the overall tract length.

\subsubsection{Diffusion and axon properties}

138 Diffusion MRI (dMRI) provides an important tool for measuring white matter microstructure 139 in vivo. Water molecules impinge on cellular membranes, intracellular organelles, 140 neurofilaments, and myelin. The diffusion displacement distribution therefore contains information about all of these structures. When the diffusion is measured in different directions, models can be applied to the data, and these models' parameters can be used to represent the white matter microstructure (53).

Axon diameter. There are several methods of acquiring and modelling $\mathrm{dMRI}$ to measure the mean axon diameter or the axon diameter distribution. Currently most of the methods are challenging since they require multi-shell high angular resolution diffusion imaging (HARDI) data, and special diffusion acquisition sequences that use either very strong and/or oscillation gradients. Furthermore, the dMRI resolution limit is very far from the resolution of axon bundles, and current methods have a limited sensitivity for small axons (54). The models used for estimating axon diameter in vivo often have many assumptions on tissue properties, that might be invalid, or require knowledge of the main orientation of fibers. Due to these limitations, in vivo measurements of axon diameter are challenging and some controversy remains regarding their feasibility (55). Nevertheless, some of the existing methods have been validated using histology and phantoms of cylinders in varying diameters $(54,56-59)$.

156 g-ratio. The voxel average g-ratio may be estimated using a model combining dMRI 157 parameters reflecting axon or fiber volume in the voxel (Fiber Volume Fraction, FVF), with 158 an MRI-myelin measurement (Myelin Volume fraction, MVF) (25). The dMRI models that 159 were previously used to calculate g-ratio include neurite orientation dispersion and density 160 imaging (NODDI) $(21,60)$, ActiveAx $(28,61)$, AxCaliber $(28,56)$, tensor fiber density (TFD) 
$(29,62)$, and tensor modeling $(25,63,64)$. It is important to note that each method has its own advantages and limitations. In many cases the limitations of these estimates come from the assumptions of their models, that might not hold true in tissue with crossing fibers and in tissue with compartments with different diffusion properties. The MVF also saw several implementations including mcDESPOT $(26,65)$, multi-exponential T2 (66-68), T2* $(69,70)$, quantitative magnetization transfer (qMT) $(25,29,63,68,71-74)$, and non-water fraction $(27,28,75)$. Most of the MVF estimates, while sensitive to myelin, are not specific. Therefore, some of these methods require calibration, and/or may provide a poor estimate of MVF in tissue that is not healthy white matter tissue $(63,70,76)$. The myelin water fraction is arguably the only specific MVF MRI measurement (67), yet it is currently hard to measure in clinical scanners which often comes with a reduced signal to noise ratio (SNR). Recent work compared the reliability of several of the different g-ratio methods and found NODDI to be more reliable than FA for the FVF estimation, where MTV and qMT were similar, with qMT having some advantage (77). Despite the limitations above, the $\mathrm{MRI}$ measurement of g-ratio was shown to provide a good estimate of the weighted average of the g-ratio in a voxel (78).

Length. Tractography algorithms use dMRI to reconstruct the white matter fiber tracts $(79,80)$. The tract reconstruction provides the pathway length which is a critical parameter for deriving conduction time from conduction velocity estimates.

180 Tract properties. Since this work aims at assessing conduction in white matter, it is necessary to characterize the white matter microstructure properties of specific relevant white matter fibers. Using tractography one can measure structural MRI measurements $(g, d)$, along the reconstructed white matter tracts, either along the core (i.e. the mean position of the streamlines' position per node), per voxel, or per streamline $(79,81,82)$.

Having performed tractography, and measuring g-ratio and axon diameter along relevant fiber tracts, one can then use these measurements in the axon model and predict the mean conduction time for an individual or groups of subjects. Additional analysis of the data might reveal more about conduction properties. For example, one can use the variance of estimated latencies within a single subject's white matter tract, and try to model the local field potential. The framework implemented in a recent study (83) allowed for a simple model that simulates LFP measures. Modelling an LFP from the MRI data allows for a more comprehensive comparison with the electrical signal: ERP width, amplitude etc. While these properties are also likely to be affected by cortical processing as well as skull properties, it is worth testing the extent to which the white matter contributes to the recorded electrical signal.

\subsection{Simulation and choice of parameters}

Several of the model parameters cannot be measured with MRI, and must be set manually. These parameters include the axon geometry, and specific electrical properties 
201 properties have been measured in rodents and primates, and are considered to be 202 conserved over species (46). Nevertheless, exceptions are possible, as evident from a 203 recent study which found that the human membrane capacitance is $0.45 \mu \mathrm{F} / \mathrm{cm}^{2}$ and not $204 \quad 0.9$ as is the case with mice (84).

205 While the electrical properties of the axon are thought to be conserved, the geometrical aspects have been shown to vary with age, disease, and possibly with learning and behavior. Therefore, a potential limitation in modeling the human white matter conduction time is that not all geometrical parameters can be measured in vivo. These parameters will have to be based on the literature. The internode length, for example, is a significant parameter (48,85-87), yet MRI measurements do not allow such information to be extracted. Fortunately, many geometrical properties of the axon share a close to linear relationship: It has been shown that $g$ can be described as a monotonic function of the axon diameter, and that the internode length is roughly a linear function of the axon diameter $(16,24,38,88)$. The latter relationship was used to reach Rushton's formulation, and it can be used explicitly when assuming an internode length for the simulation.

We explored the conduction velocity as calculated with the simulation, for a range of values for axon diameter $(d)$ and fiber diameter $(D)$ ). We chose values that were found for myelinated axon in the human central nervous system (89), with values ranging from 0.2 to $5 \mu \mathrm{m}$ for both $d$ and $D$ The internode length was calculated as a linear function of the fiber diameter, as found in the anterior medullary velum of the adult rat : $L=117+30 \cdot D$ (90). The specific membrane capacitance was set to $0.45 \mu F / \mathrm{cm}^{2}$ (84). The rest of the (electrical) properties were fixed as described in table 1 of Lorena et al,'s paper (52). It can be seen in Figure 1a that the larger the fiber diameter $(D)$, the faster it will conduct. Furthermore, for a given fiber diameter, the conduction velocity as a function of axon diameter $(d)$ has a maximum around $g=0.6$, as shown by Rushton. The intuition for this is the following: given a constant fiber diameter, dramatically decreasing $g$ means a very small axon diameter, thus reducing intra-axonal conductivity resulting in slower conduction. However, dramatically increasing $g$ will reduce the leak resistance (46), which will result in slower conduction as well. Therefore, the optimal $g$ should lie somewhere in the middle, and Rushton's analysis found it to be 0.6. As mentioned in the Introduction, Rushton's analysis considered the peripheral nervous system. More recent theoretical study focused on the rat central nervous system (19), and found that given volume and energy consumption constraints, the optimal $g$ should be approximately 0.77 .

234 In the simulation described above, we varied the g-ratio and the axon diameter. However, 235 since in our MRI dataset (as in many others) we cannot calculate an estimate of axon 236 diameter, we test whether measuring $g$ alone will be meaningful. To calculate conduction velocity with no estimate of axon diameter, we must first choose and fix the axon diameter for all tracts and subjects. The choice of axon diameter should be informed by known histological measurements, and the fact that MRI measurements, and the g-ratio in particular, are volume-sensitive (91). Aboitiz (89) measured the axon diameter distributions in the human corpus callosum. The results show that the axon diameter distributions are mostly centered around values close to $1 \mu \mathrm{m}$, with very few axons as large 
243 as $5 \mu \mathrm{m}$ in diameter. To approach the volume-sensitive g-ratio MRI measurement, we 244 calculated a weighted average of the distributions derived by Aboitiz (weighted by axon 245 diameter to give more weight to larger diameters). We find that the weighted average of 246 the callosal splenium, mid-body and genu are 1.72, 2.81 and $1.3 \mu \mathrm{m}$, respectively. This 247 anatomical segmentation of the corpus callosum corresponds to the functional 248 segmentation of the corpus callosum to its occipital, motor and frontal tracts, respectively.

249 We predicted velocities of axons with a radius of $1.72,2.81$ and $1.3 \mu \mathrm{m}$ (the weighted 250 average of the callosal axons), given a biologically relevant range of $g$, between 0.7 and 2510.85 (Fig. 1b). Next, we estimate the theoretical latency of these axons if they were $10 \mathrm{~cm}$ 252 long, about the length of motor callosal fibers (Fig. 1c). We find that using our estimates 253 of volume-weighted average of axon diameter in the numerical simulation leads to callosal 254 delays around 7-25 ms. This range is close to that found in electrophysiological studies 255 (92-96), and using response-time differences $(33,97)$. For the following sections, we will 256 use an axon diameter of 1.72, 2.81 and $1.3 \mu \mathrm{m}$, for the occipital, motor and frontal tracts, 257 respectively. It is noteworthy to mention that a smaller axon diameter creates longer 258 delays, and it also increases the effect of $g$ on the conduction time estimate (because the 259 velocity is much smaller). Since the motor tract has the largest weighted average of axon 260 diameter, it will most likely have the shortest conduction delay. The conduction delay time 261 in the motor fiber tract won't be the shortest only if the fiber is substantially longer, or has 262 a dramatically lower g-ratio value. Neither of which are to be expected. For further testing 263 regarding the axon diameter distribution's effect on the conduction see discussion and 264 Fgure 6.

265

(a)

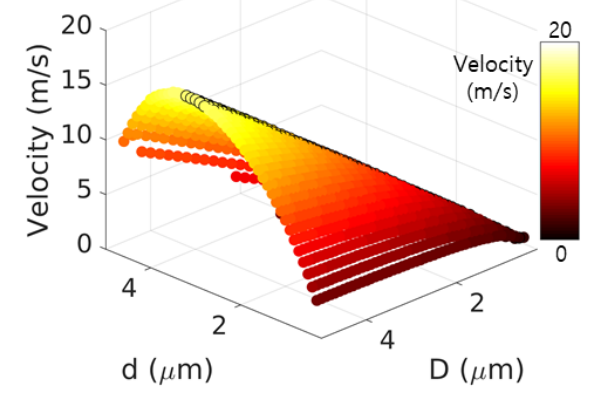

(b)

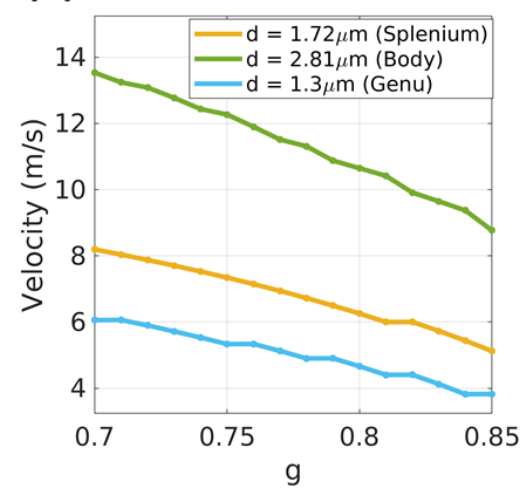

(c)

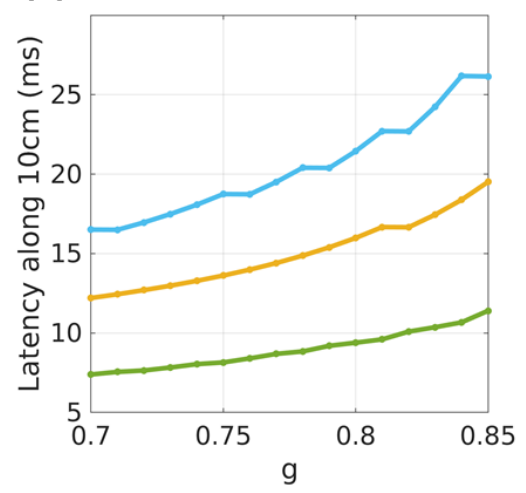

Figure 1: Simulating conduction as a function of axonal properties. A numerical simulation (51) for estimating the conduction velocity. (a) Conduction velocity plotted as a function of axon (d) and fiber (axon + myelin) diameter (D), peak velocity can be found around g-ratio=0.6, marked with black circles. (b) Conduction velocity as a function of a small biological range of $g$, plotted for three values of axon diameter $(1.72,2.81,1.3 \mu \mathrm{m})$, corresponding to the volume-weighted averages of the axon diameter distributions measured by Aboitiz et al., (1992) in the callosal splenium, mid-body and genu (respectively). (c) Conduction delay for the velocities in (b) and a distance of $10 \mathrm{~cm}$. The delays for the larger axons are around 5-25ms, similar to inter hemispheric transfer time measured with EEG. The difference in axon diameter between the tracts leads to a large difference in latency. 


\section{Methods}

267 Having established a framework aimed at relating qMRI data to a biophysical model of 268 conduction, we tested whether it would provide sound estimates of conduction latency 269 along white matter fibers. We calculated the predicted latency of signal transfer along 270 callosal fibers in two subject groups that are expected to have variance between them.

\section{$271 \quad 3.1$ Participants}

272 The MRI measurements were performed on 21 young adults (aged $27 \pm 2.1$ years, 9 273 females), and 17 older adults (aged $67.4 \pm 6$ years, 5 females). The Helsinki Ethics 274 Committee of Hadassah Hospital, Jerusalem, Israel approved the experimental 275 procedure. Written informed consent was obtained from each participant prior to the 276 procedure.

\section{3.2. MRI Acquisition}

278 Data was collected on a 3T Siemens MAGNETOM Skyra scanner equipped with a 32279 channel head receive-only coil at the ELSC neuroimaging unit at the Hebrew University.

280 Quantitative T1, \& MTV mapping: 3D Spoiled gradient (Flash) echo images were acquired with different flip angles $\left(\alpha=4^{\circ}, 10^{\circ}, 20^{\circ}\right.$ and $\left.30^{\circ}\right)$, TE/TR $=3.34 / 19 \mathrm{~ms}$. The scan resolution was $1 \mathrm{~mm}$ isotropic. For calibration, we acquired an additional spin-echo inversion recovery scan with an echo-planar imaging (EPI) read-out (SEIR-epi). This scan was done with a slab-inversion pulse and spatial-spectral fat suppression. For SEIR-epi, the TE/TR was 49/2920 ms. TI were $200,400,1,200$, and 2,400 ms. We used 2-mm inplane resolution with a slice thickness of $3 \mathrm{~mm}$. Both the flash and the SEIR-epi scans were performed using 2× GRAPPA acceleration.

Diffusion mapping: Whole-brain DWI measurements were performed using a diffusionweighted spin-echo EPI sequence, accelerated by a factor of 2 (GRAPPA), with isotropic $1.5-\mathrm{mm}$ resolution. The acquisition included 2 diffusion-weightings, one with 32 noncollinear directions $\left(b\right.$-value $\left.=1000 \mathrm{~s} / \mathrm{mm}^{2}\right)$ and a second with 64 non-collinear directions $\left(b\right.$-value $\left.=2000 \mathrm{~s} / \mathrm{mm}^{2}\right)$. The scan also contained 8 volumes without diffusion weighting $(b$-value $=0)$. In addition, we collected one scan with six non-diffusionweighted volumes and a reversed phase encoding direction (posterior-anterior) to correct for echo-planar imaging distortions due to inhomogeneities in the magnetic field.

\subsection{Estimation of qMRI parameters}

297 Quantitative MTV \& T1 mapping: Whole-brain MTV and T1 maps, together with bias 298 correction maps of B1+ and B1-, were computed as described in $(75,98)$. In short, 299 unbiased T1 maps were calculated using the variable flip angles which were corrected for 300 B1 excite inhomogeneity using the unbiased SEIR data (99). Next, the T1 maps were used to calculate unbiased proton density (PD) maps. To separate PD from receive-coil 302 inhomogeneity, we assume smooth coil functions and use a biophysical regularization, 303 which finds local linear relationships between 1/T1 and PD. This method was found to be 
304 effective and robust to noise (100). The PD was normalized according to values in CSF305 only voxels in the ventricles, to produce water-fraction (WF) maps. The MTV maps were 306 then calculated as 1-WF. The analysis pipeline for producing unbiased T1 and MTV maps 307 is an open-source MATLAB code (available at https://github.com/mezera/mrQ).

308 Diffusion parameter estimation: Diffusion analysis was done using the FSL toolbox (101); 309 Susceptibility and eddy current induced distortions were corrected using the reverse 310 phase-encode data, with the eddy and topup commands $(102,103)$. The corrected, 311 unwarped data were aligned to the imaging space of the MTV map using FSL's Flirt rigid312 body alignment (104,105). Furthermore, the data was fit with the Neurite Orientation and 313 Dispersion Diffusion Imaging (NODDI) model using the AMICO toolbox $(60,106)$.

314 g-ratio: In the corpus callosum, the g-ratio was calculated with the following formula, using 3152 different measurements: $g=\sqrt{1-M V F / F V F}$. MVF was estimated using MTV $(27,28)$. 316 The FVF was derived from MTV and the isotropic $\left(V_{i s o}\right)$ and intracellular $\left(V_{i c}\right)$ volume 317 fractions estimated from NODDI (21): $F V F=M T V+(1-M T V)\left(1-V_{i s o}\right) V_{i c}$. Supp. Fig. 3181 shows examples of the qMRI maps used in this study ( $V_{i c}, V_{i s o}, M T V$ and g-ratio) for 3 319 younger and 3 older subjects.

320 Tractography: We performed whole-brain anatomically constrained tractography using the 321 mrTrix software (80). The white matter tracts in corpus callosum were then segmented 322 using the Automated Fiber Quantification (AFQ) toolbox (79). We used this software to 323 track three major callosal tracts connecting the two hemispheres: occipital, motor, and 324 anterior-frontal. We used the AFQ software to sample $g$ along the tract core, and create a 325 tract profile of $g$ along each tract. The tract profiles are calculated as a weighted sum of 326 each streamline's $g$ value at a given node, where each streamline is weighted based on 327 its Mahalanobis distance from the core of the tract. The result is a vector of 100 equidistant 328 measurements of g-ratio along the trajectory of each callosal tract. The profile can be 329 average over all or some of the nodes to produce a single value for each tract. 330 Furthermore, we use code from the vistasoft git repository to estimate a median $g$ value

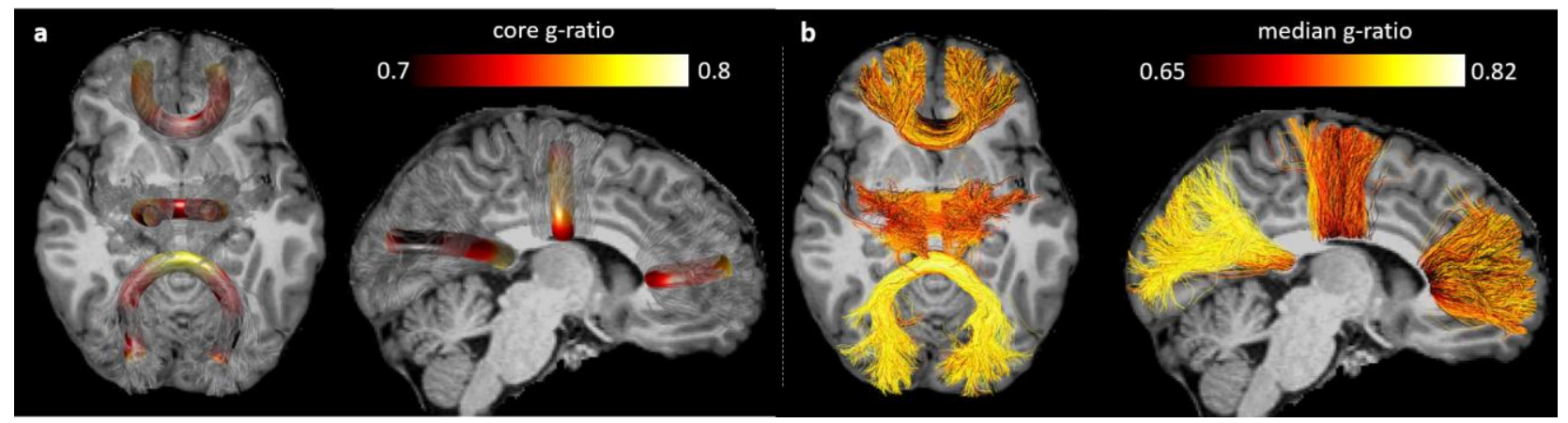

Figure 2: sampling g-ratio along a fiber tract. The g-ratio values along 3 callosal tracts of a single subject: the occipital, motor, and anterior-frontal callosal tracts. The g-ratio can either be sampled (a) along the tract core, or (b) for each streamline separately. The two methods sample the space differently, but both show a variance between and within fiber tracts. The average values of $g$-ratio along the tract core across subjects can be seen in Supp. Fig. 2 
331 for each streamline. The median value of each streamline is calculated over the 20 nodes

332 around the midsagittal plane. Fig.2 presents an example of the two types of sampling 333 strategies, and Supp. Fig. 2 shows the trajectory of g-ratio along the fiber tracts, averaged 334 across subjects.

335 One young subject was removed from the analysis due to unsuccessful unwarping of the 336 diffusion data. One older subject was removed from the analysis due to extremely sparse 337 tractography results in the occipital cortex. The rest of the subjects (20 young and 16 older 338 subjects) were included in all analyses.

\section{3.4. Latency estimation}

340 To test whether we predict a delay in callosal conduction time with age we first calculate the mean of the $g$-weighted MRI measurement along the three callosal tracts. The mean

342 was calculated over the 20 medial nodes of the tract/streamline (for more details on the 343 effect of the choice of node number see Supp. Fig. 2). We incorporate the mean $g$ values in the simulation to estimate conduction velocity along the different fiber groups. The model parameters were identical in all simulations with the following exceptions: the gratio values were taken from the MRI measurements, the axon diameter was different for each callosal tract (but the same across subjects) and the inter-node length was calculated as a function of g-ratio and axon diameter (see Theory section). The latency of each tract was calculated by dividing the tract length by its velocity. We hypothesize demyelination with age, and a consequent increase in g-ratio, decrease in conduction velocity, and increase in conduction time. Therefore, we used a one-tailed t-test to compare younger and older subjects' values for each fiber tract, in each of these measures (g-ratio, velocity, and conduction delay).

354 Finally, we used the calculated latency of each streamline to simulate an LFP signal for each fiber tract. The simulation was adapted from the analysis in (83). The LFP is simulated as the sum of the time-integrated input of a population of neurons. Each neuron receives its input from one streamline. An example of this process is presented in Supp. Fig. 3. First, the median g-ratio value of each streamline is incorporated in the simulation to produce conduction velocity and together with its length, we calculate its conduction delay. The delay times of all streamlines within a single tract, are used as input, which is modeled as Gaussian noise, with a delta function of the corresponding latency of the streamline (Supp. Fig. 3a). Simulating a leaky neuron, the input is integrated over time (Supp. Fig. 3b). Finally, the sum over the streamlines' simulated time series is treated as the LFP signal. To allow for a comparison between tracts that differ in the number of streamlines, the signal is normalized to have a peak amplitude of 1 [a.u] (Supp, Fig. 3c). The simulated LFP signal has a large peak similar to an event related potential (ERP), and we extracted the peak time and the full width half maximum of the ERP. We used a onetailed t-test to compare the LFP peak time and two-tailed t-test to compare the width of younger and older subjects' simulated LFP signal for each fiber tract. Software for the 370 analysis in this study can be found at GitHub [link]. 
372 Finally, we considered the effect of the known axon diameter distributions on the 373 conduction velocity, and in particular its effect on differences between regions. We 374 reproduced the histograms of the axon diameters in the splenium, genu and mid-body of 375 the corpus callosum, as presented in Figure 4 in Aboitiz et al. (89). To model conduction 376 based on these distributions we performed the same simulations described in the theory 377 and methods section (sections 2.3 and 3.4), with one exception: we estimated the g-ratio 378 for each value of axon diameter using $g=0.22 \cdot \log (d)+0.506$ (107). This relationship 379 was derived from axons of the peripheral nervous system, for axons diameter between $380 \quad 0.5 \mu \mathrm{m}$ and $4 \mu \mathrm{m}$. Nevertheless, similar trends can be observed in the CNS (78). From the axon diameter and g-ratio distribution we estimated the distribution of velocities of the three regions. We then calculate latency distributions either for a fixed length of $10 \mathrm{~m}$ or for the mean tract lengths that we measured using the tractography results (section 3.3). Finally, we simulate the LFP of each region, using the distribution of latencies as input to the simulation (sections 2.3 and 3.4).

\section{4. Results}

387 To test whether the $g$-weighted MRI measurement can be incorporated in our framework 388 for estimating changes in white matter conduction with age, we first evaluated the $g$ values in the corpus callosum of younger and older subjects. Figure 3a shows the average $g$ over the 20 medial nodes of the tract core, for each age group and fiber tract (Supp. Fig. 2 shows the effect of the number of nodes taken around the midline). We find that older subjects have higher $g$ values compared with younger subjects only in the motor callosal tract ( $p=0.03, t_{32.6}=-1.95$, uncorrected for the three comparisons). We also found differences between the callosal regions (see Fig. 5). However, these differences are highly affected by the distance from the mid-sagittal plane suggesting that the calculated g-ratio is sensitive to partial volume effect between white matter tracts (Supp. Fig. 2).

397 We incorporated the $g$-weighted measurements in the simulation to calculate conduction velocities (Fig. 3b). The simulations (Fig. 1b) reveal the dependence of the conduction velocity on g-ratio, (given a fixed axon diameter). The increase of g-ratio with age in the motor callosal region leads to a small but significant decrease of the simulated conduction velocity with age ( $p=0.02, t_{33}=-2.13$, uncorrected for the three comparison). Next, we combined the velocity with the fiber tract lengths (Fig. 3c), to calculate conduction time (Fig. 3d). We found no significant differences between the predicted latencies of younger 404 and older subjects, in all areas.

405 Next, we used the tractography streamlines to predict more conduction properties. We 406 treated each streamline as a separate representative of underlying axonal properties 407 (instead of taking the tract core's properties to reflect a single value for all streamlines). 408 This sampling approach creates a distribution of $g$ values. Each streamline has its own 409 length and g-ratio, thereby resulting in a distribution of estimated latencies. As is for the 410 core (Fig. 3), $g$ was sampled along the 20 medial nodes of each streamline, and the 411 median of this sample was used. The distribution of latencies can be used for simulation 

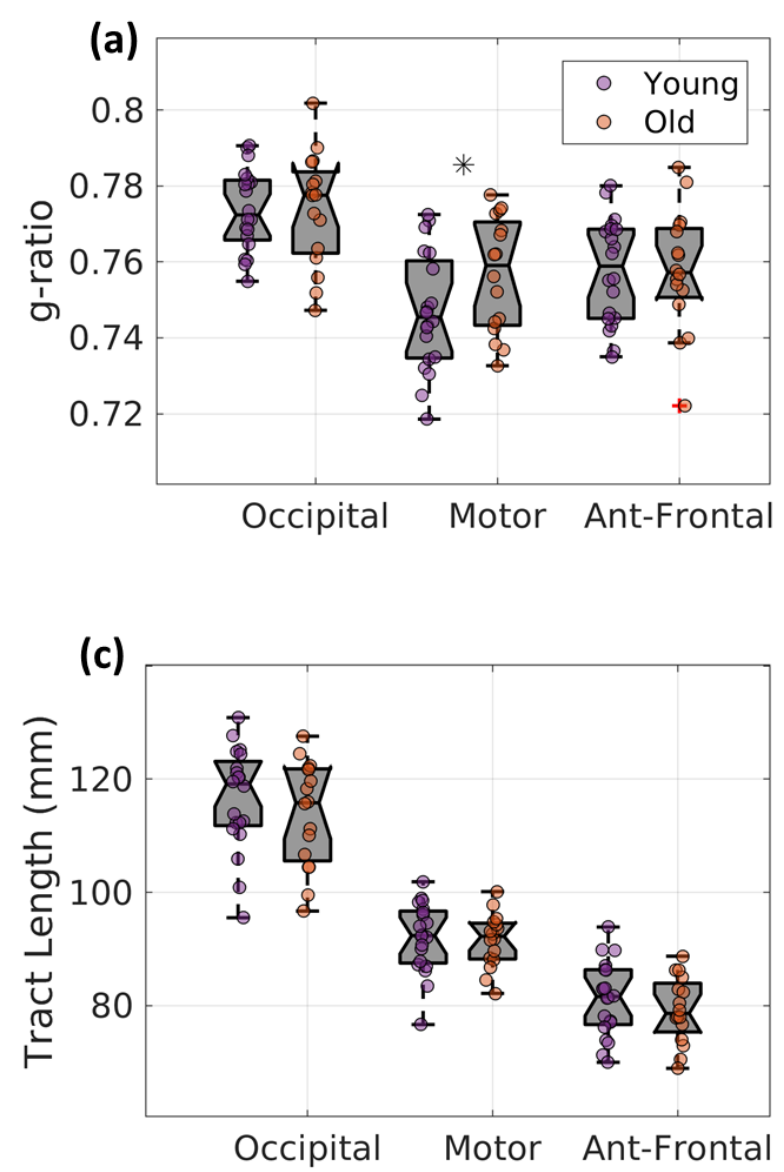
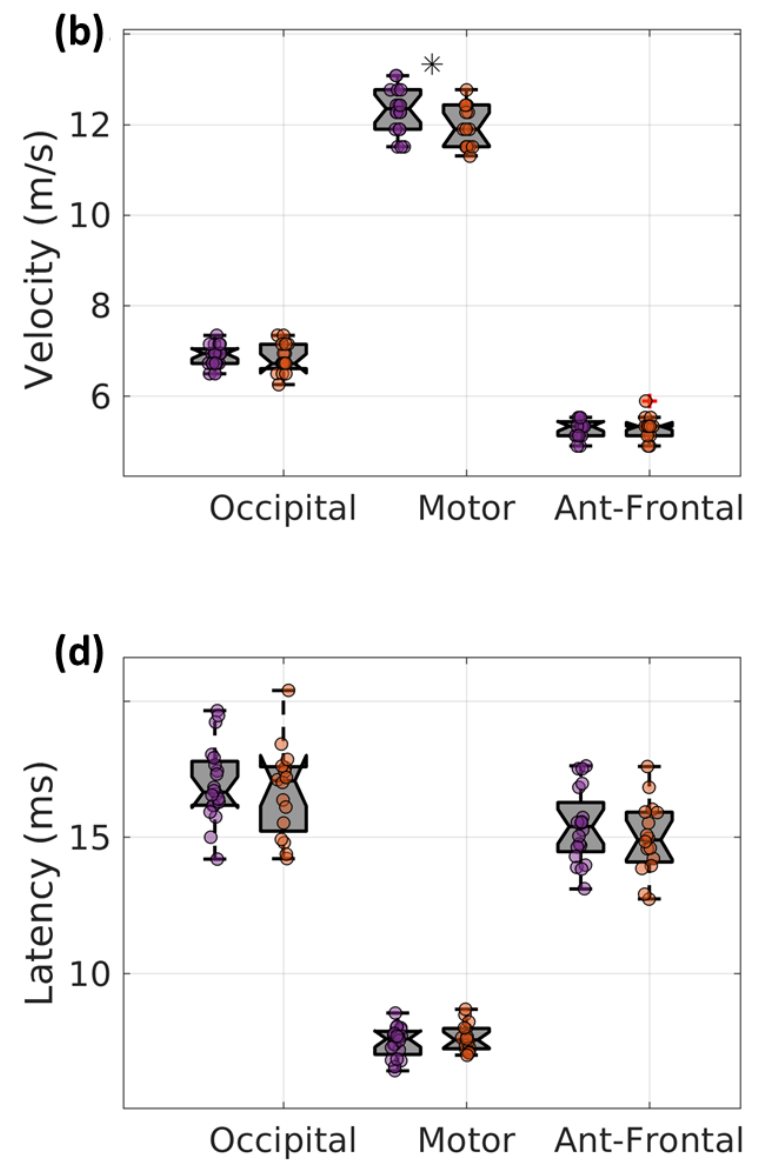

Figure 3: Predicting conduction velocity and latency. (a) g-ratio calculated with MTV and NODDI, in three callosal tracts. In the motor callosal region the g-ratio is higher in older (orange) compared with younger (purple) subjects $\left(p=0.03, t_{32.6}=-1.96\right)$. (b) The g-ratio values were used to numerically simulate the conduction velocity along each tract (51). We find that the model predicts a decrease in conduction velocity with age in the motor callosal region $\left(p=0.02, t_{33}=2.13\right.$ ). However, there are no significant differences between the groups' (c) tract length or (d) the conduction delays derived from the tract length and the tract conduction velocity.

412 of an LFP signal (shown in Fig. 4a). The peak time of the LFP is highly correlated with the 413 latency calculated using the tract core $\left(R^{2}=0.97\right)$, but it is not identical as it is sensitive 414 to the variance across the tract (Fig. 4b). Comparing the latencies between the age groups 415 (Fig. 4c), we found a delay of conduction latency with age in the motor callosal region ( $p=$ $4160.03, t_{30.9}=-1.92$, uncorrected for the three comparison).

417 The LFP analysis also allows us to compare the variance in the data using the signal width. 418 Figure $4 d$ shows the comparison of the full-width half-maximum of the simulated LFP 419 signal between the age groups. Interestingly, this comparison of ERP width between 420 younger and older subjects also reveals a significant difference with age in the callosal 421 fibers connecting the occipital cortices $\left(p=0.02, t_{28.9}=-2.13\right.$, uncorrected for the three 422 comparisons), suggesting a higher variance within subject, with age. All comparisons 423 between younger and older subjects are not significant after an FDR correction. 
424 Finally, both the core analysis and the LFP simulation predict that the fastest fibers will be 425 in the motor tract of the corpus callosum, with the occipital fibers as much as $10 \mathrm{~ms}$ slower. 426 To test whether this effect depends on the summary statistic we calculated from the axon 427 diameter distribution, we used the entire distribution to simulate the latency of each 428 callosal regions. The axon diameter distribution shown in Fig. 5a. are adapted from the
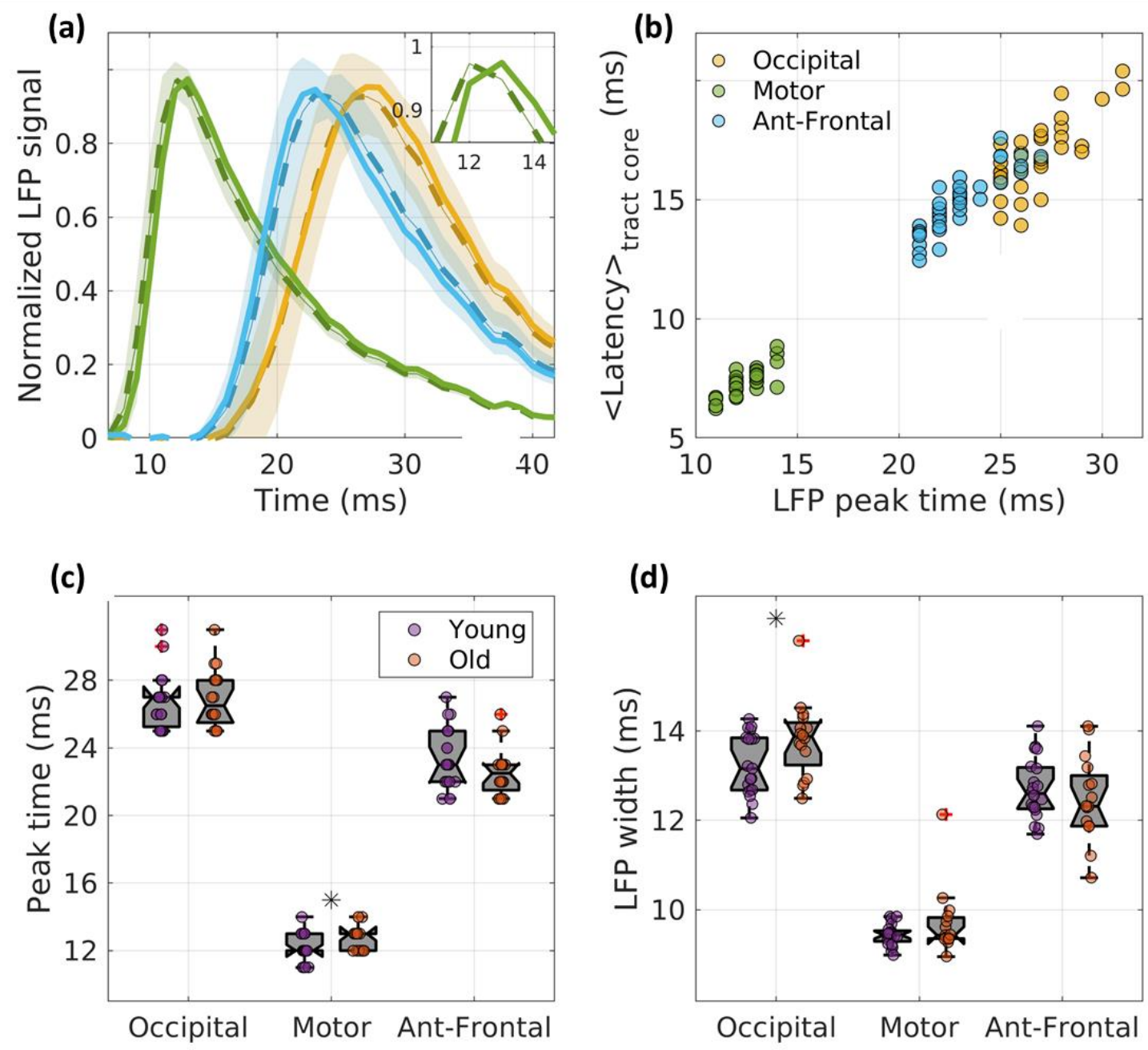

Figure 4: LFP estimates. The g-ratio and length of each streamline was used to calculate the conduction latency. The distribution of latencies for each tract was then used to simulate an LFP signal. (a) Simulated LFP for young (solid line) and old (dashed line) in the three fiber tracts. The shaded area is the STD across subjects. The inset zooms in the peak of the LFP for the motor tract of younger and older subjects. (b) A comparison of the LFP peak time with the mean latency of the tract core $\left(R^{2}=0.97\right)$. The values are not identical but the overall variance is maintained. Group comparison of the LFP peak time (c) and width (d) reveal different effects than those calculated with the core. The LFP peak time is delayed in the motor callosal region of older subjects $\left(p=0.03, t_{30.9}=-1.92\right)$. The LFP width of older subjects is significantly larger in the occipital tract $\left(p=0.02, t_{28.9}=-2.13\right)$. 

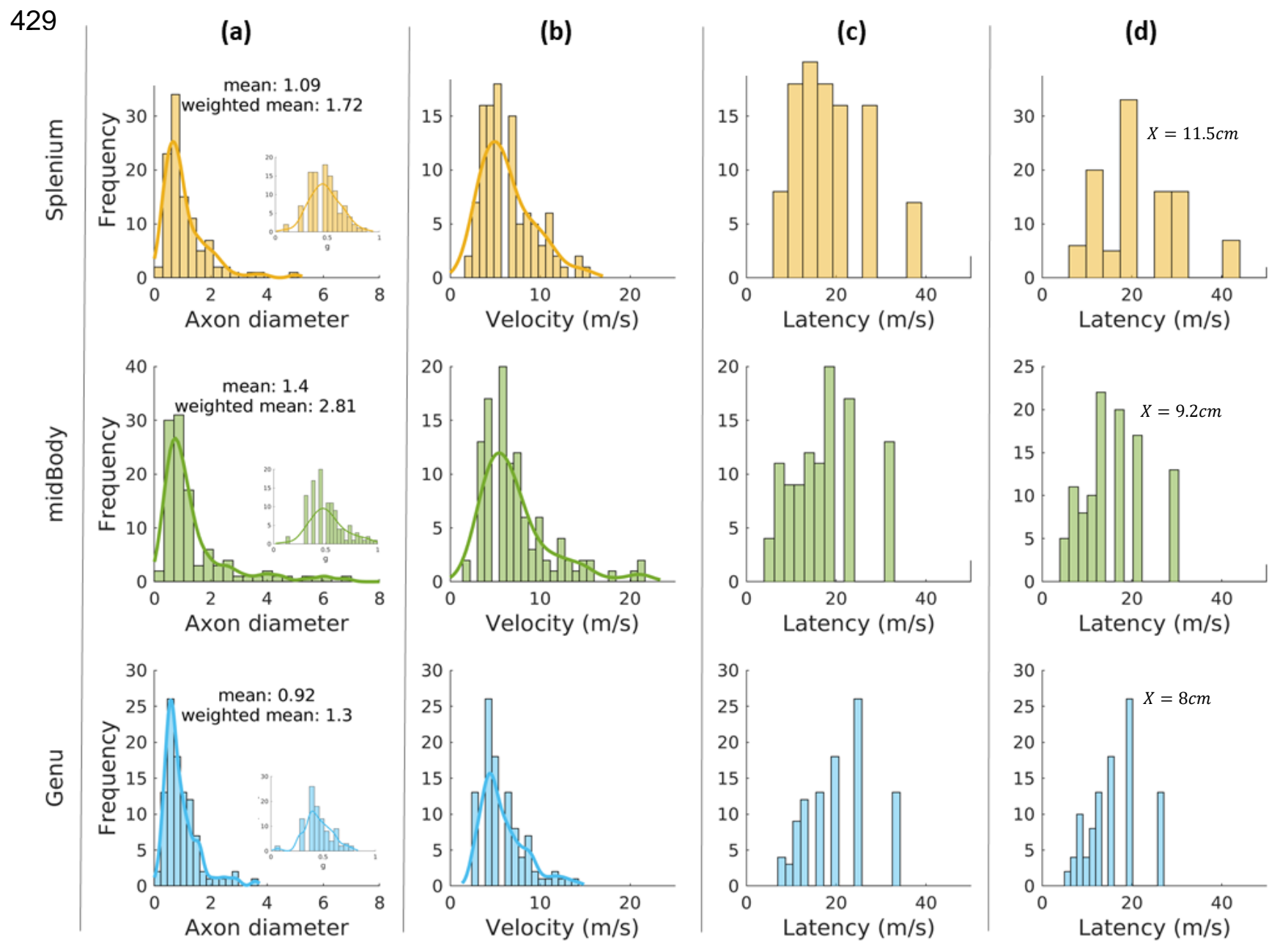

(e)
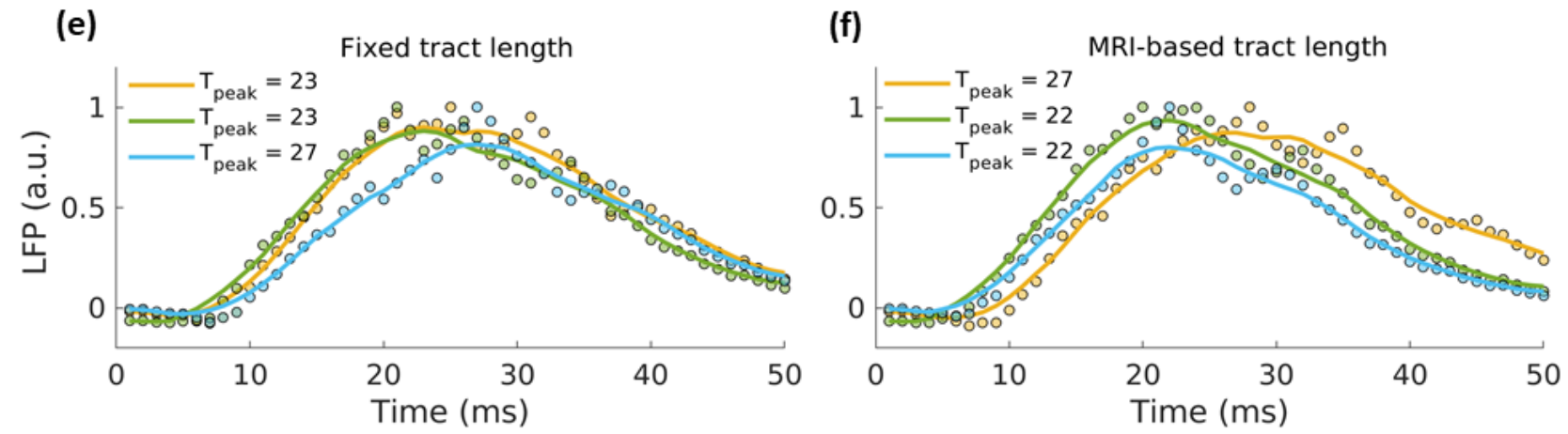

Figure 5: Conduction of axon diameter distribution in the corpus callosum. (a) a reproduction of the histograms of the axon diameters in the splenium genu and mid-body of the corpus callosum, as presented in Figure 4 in Aboitiz et al. (1992). (b) The distribution of simulated velocities of the three regions, revealing a larger fraction of fast axons in the mid-body. (c) The distribution of latencies given a $10 \mathrm{~cm}$ long axon. (d) The distribution of latencies given the tract lengths calculated from tractography: $11.5,9.2$ and $8 \mathrm{~cm}$ for the splenium, mid-body, and genu, respectively (e) Simulation of the LFP given the latencies in (c), suggests a longer delay in genu fibers. (f) Simulation the LFP of each region, given the velocities in (d) and the tract lengths from the tractography data. The results suggest longer conduction delays in the splenium. 
distribution of the genu, mid-body and splenium, plotted in Fig.4 of Aboitiz's study (89). Figure $5 \mathrm{~b}$ displays the distributions of conduction velocities that were simulated from the axon diameter distribution, and their corresponding g-ratio distributions (insets in Fig. 5a). The simulation derives distributions with similar peaks for the three regions, but a larger fraction of 'fast axons' for the mid body segment. We simulate the LFP signal that would arise from the distribution of latencies for a $10 \mathrm{~cm}$ long axon (Fig. 5ce). We find the genu display the most delayed peak. Next, we plot the LFP and latency distribution given the tract lengths calculated from tractography (Fig. 5df). The average tract length for occipital $(11.5 \mathrm{~cm})$, motor $(9.2 \mathrm{~cm})$ and anterior-frontal $(8 \mathrm{~cm})$ fiber tracts were used for the LFP simulation of the splenium, mid-body, and genu, respectively. Using the tractography fibers length estimates we find the splenium is estimated to have the most delayed response, while the genu and motor body have similar conduction delays. This result highlights the potential differences between conduction velocity and delay time.

\section{Discussion}

444 In this study we provide a biophysical framework which relates qMRI measurements to white matter signal conduction, in an attempt to provide a link between structure features of axons and electrophysiology. We used this framework to test whether the MRI measurement of $g$-ratio can be used to model changes in conduction properties with age. We estimated the conduction velocity and conduction time in the corpus callosum of healthy human subjects. We found little to no significant difference between the young and old subject in the $g$-weighted measurement, and in conduction estimates in the occipital frontal and motor callosal fibers.

It would have been of great interest to evaluate the sex differences in changes in conduction with age. Unfortunately, we do not have enough data to support such an analysis. Nevertheless, in a previous study we measured g-ratio in the corpus callosum in 80 people of ages 8 to 81 years old. We found no differences in the males and females. Similar results were found by Cercignani et al. (74), using a different dataset, and slightly different measurements to estimate g-ratio.

The biophysical interpretation of the qMRI measurements relates them to properties of signal conduction along a myelinated axon. That is, estimating conduction velocity as a function of axon properties (axonal diameter, myelin thickness, and fiber length). There are other models that could have been used here. For example, given that the conduction velocity is indeed mostly affected by the geometric characteristics of the internode (rather than the node), one can reduce the conduction velocity of the axon to the conduction velocity of the internode. Assuming a semi-infinite cylinder, the conduction velocity is a function of the membrane time constant $(\tau)$ and length constant $(\lambda)(108,109) \theta=2 \lambda / \tau$. This solution describes unmyelinated axons better than myelinated ones, but it is convenient since it doesn't require a full simulation of the axon propagation. Different models will produce different predictions of conduction latency, but unless one chooses a model that is based on a very different set of assumptions of the axon biophysics, we do not expect it will lead to a great difference in the results. 
471 When applying a biophysical model to MRI data, it is necessary to extract summary 472 statistics of the microstructure parameters. One possibility is to use the voxels in a certain 473 region (e.g. their mean). To sample white matter pathways, it is advantageous to rely on 474 the tractography results, and either use the tract core values, or obtain the characteristic 475 value for each streamline. While the tract core values are probably less susceptible to 476 partial volume effects, using the streamlines allows an interesting and potentially 477 informative sampling of the space.

478 Using the streamlines as a way to estimate a distribution of axonal values is a very coarse reduction of the anatomy. Whether or not it is valid depends on the homogeneity of the axons in space within a fiber tract. Future testing is needed to decide if ERP characteristics such as latency or width are helpful when interpreting the structural MRI data. To our knowledge, there is not enough evidence today to determine whether it is helpful to use such analysis. Nevertheless, in our data, the LFP analysis predicts group differences both in the peak time and importantly, in the width of the ERP, which is not available when using the tract core values. The difference in ERP width suggests that the LFP simulation captures somewhat different information in the data than using the tract core values.

487 Using the LFP analysis, we find a significant difference in conduction delays in the motor region (Fig. 4c). However, it is a very small difference ( 2ms), and it is not replicated using the core values (Fig. 3c), suggesting that this result is model-dependent. The existing literature on callosal delays does suggest an elongation of signal transfer time with age $(33,34)$. There are several possible explanations for this discrepancy. First, given that there is an increase in inter-hemispheric transfer time (IHTT) with age, it is possible that the increase is a result of cortical processing and not changes in white matter conduction. Second, as previously discussed (27), the myelin measure used here (MTV) is sensitive, but not specific to myelin. Therefore, as the tissue undergoes changes in composition with age, it is possible that our $g$-weighted measurement is becoming less specific to myelin (i.e., affected by the contribution of non-myelin tissue component). On a similar note, it is possible that the single axon model is too coarse, and it might be necessary to formulate a model that considers the entire axonal populations that affects the MRI measures. Furthermore, while the fibers in the corpus callosum are considered to be coherent in orientation, there is evidence suggesting fine dispersion of the fiber orientation (110). Such variation in the fiber orientation could both introduce error to the estimation of the NODDI

504 Third, the g-ratio might not capture enough of the variance that is essential for the 505 calculation of conduction velocity. If the tissue changes with age, it is unlikely that only one 506 aspect of the tissue changes. Unfortunately, the biophysical model is relying on structural 507 parameters that are not available in vivo. Therefore, it is possible that one of the 508 parameters we kept constant in the calculation of conduction velocity, in fact changes with 509 age. Simulation studies have shown that the biggest factor of conduction velocity is the 510 geometry of the internode $(42,48,111)$. The internode length, for example, is an important 511 factor that may be modulated and affect conduction velocity $(86,87)$. While we do not have 
512 access to it, it was found to be correlated with axon diameter, and we use this relationship 513 to reduce the number of fixed parameters.

514 It is well established that the axon diameter is a very important determinant of conduction 515 velocity $(18,48)$, and this could have further implication for neural computation (112). 516 Furthermore, studies have shown that the axon diameter distributions are related to 517 information transfer rate and its effectiveness (113,114). Therefore, by fixing the axon 518 diameter we are probably losing information $(92,93,95,96)$. A second consequence of 519 choosing a certain axon diameter (and internode length), is that it changes the effect of $\mathrm{g}$ 520 ratio on the conduction velocity. The lower the axon diameter, the less the g-ratio will affect 521 the velocity prediction. Thus, when calculating the latency (rather than velocity), choosing 522 a small axon diameter will weight the tract lengths more strongly. Our results highlight the 523 significance of the ongoing efforts in the qMRI community to improve and simplify in vivo 524 measurement of axon diameter (e.g. 24,49,50,52,102).

525 Due to the importance of the axon diameter we chose empirically based values, from post mortem histology of human corpus callosum. We chose to fix the axon diameter in the occipital, motor and anterior-frontal tracts of the corpus callosum, to be 1.72, 2.81 and 1.3 $\mu m$, respectively. The difference in axon diameter values leads to large differences in the conduction estimates between tracts. We found that using the empirically based values of axon diameter indeed produces a range of conduction latencies which are close to those found in the literature. Nevertheless, regarding the g-ratio measurement of the different tracts, we find that in this study the between-tract comparisons largely depend on our sampling strategy. In Supp. Fig. 2 it's clear that the g-ratio changes drastically along the occipital tract. It is possible that the variability in g-ratio along the occipital tract is due to the other fiber tracts it crosses paths with. The optic radiation, which is adjacent to the splenium fibers (117) and could therefore lead to partial volume effects, is known to be highly myelinated (118) causing a large decrease in g-ratio. The idea that the trend of $\mathrm{g}$ ratio along the occipital callosal fiber is influenced by adjacent fibers is in line with the fact that it is similar between younger and older subjects (Supp. Fig. 2).

540 Our results point to interesting differences between the three callosal tracts used in this 541 study. We find the g-ratio is the highest in the occipital tract, replicating our previous study 542 (119), as well as other studies $(20,29)$. To estimate the conduction velocity we fix the axon diameter using the weighted average of the axon diameter distributions measured by Aboitiz et al. (89). The mid-body, corresponding to the motor tract, has a large fraction of large axon. This in turn, leads to the faster estimates of conduction velocity and shorter delay time (Fig. 4bd, and Fig. 5c.). Using the entire axon diameter distribution to estimate the conduction properties of the tracts (Fig. 6), we find that given a constant tract length, the splenium and mid-body have very similar latencies, and the genu has the largest delay (Fig. 6e). However, taking into account the tract lengths as measured with dMRI, we find the genu and mid-body display similar latency, while the splenium is the slowest (Fig. 6f). Interestingly, in Fig. 4, we also find that the velocity might change slightly with age, yet the latency does not. Highlighting the fact that while the velocities along the corpus callosum may vary, the latencies could show a different trend as function of the tract length. 
554 The analysis incorporating the axon diameter distributions estimates the slowest 555 conduction will be in the splenium of the corpus callosum, containing occipital fibers (Fig. 556 6ef). We find the same results using the weighted average of the distributions and the $\mathrm{g}$ 557 ratio measured using MRI (Fig. 4d, and Fig. 5c). Nevertheless, while the former analysis 558 finds similar latencies for the genu and mid-body, the latter estimates the mid-body to have 559 faster conducting fibers. The discrepancy is likely due to the difference between using the 560 entire distribution, and using a summary statistic to represent it: the large fraction of fast 561 axons in the mid-body increases its weighted average dramatically, leading to large differences in the velocity estimates of the three tracts. However, when using the entire axon diameter distribution, we derive distributions of velocities with fairly similar peaks, leading to closer estimated of LFP peak time. Other differences originate from the way the latency distributions are constructed. The latency distributions (from which the LFP is simulated) derived in Fig. 5 ac reflects the variance of g-ratio within tract, whereas in Fig. 6 they represent the variance of axon diameters, measured with histology. One last important distinction is that in the axon diameter distribution analysis we choose g-ratio as a function of the axon diameter. This causes larger axons to have larger g-ratio, meaning less myelin. This could decrease the differences between the tracts. Note that while one can assume a certain g-ratio per axon diameter, the opposite deduction is harder to make. This is due to the shape of their relationship: a large range of axon diameter can have similar g-ratios. Furthermore, we do not know how the axon diameter distribution changes with age. These results suggest that more precise histological analysis of axon diameter and g-ratio in the corpus callosum body is needed.

576 Finally, the literature describing an increase in IHTT with age, did not measure callosal conduction directly, but instead used indirect behavioral measures such as response time. Therefore, it is possible that a more direct estimation of callosal delays will reveal that they are indeed stable with age. The conduction delays can be estimated from the evoked response potential which is measured in vivo with methods with high temporal resolution (e.g., ECoG, EEG and MEG). The M/EEG signal represents a conglomeration of many different neural sources of activity (120). Embedded in the signal are the neural responses associated with specific sensory, cognitive, and motor events. Conduction along the corpus callosum can be accessed by presenting subjects with unilateral stimuli (tactile or visual), since such stimuli are first processed in the contralateral hemisphere, and the information is then transferred to the ipsilateral hemisphere via the corpus callosum. By measuring the same early ERP components (e.g., parietal P100) from the different hemispheres, and computing the difference between their time of occurrence, one can estimate the IHTT - the time it took the signal to travel from one hemisphere to the other,

591 We tested the power of a $g$-weighted MRI measurement to predict conduction delays with 592 age, and we propose that future studies would benefit from testing the framework by 593 measuring conduction delays and structural MRI together. It should be noted, however, 594 that the structural MRI measurements cannot be expected to fully explain the variance in 595 the ERP latency. Of course, a higher resolution and SNR might improve the prediction. 596 Nevertheless, predicting conduction delays can also have other limiting factors. Currently 
597 it is impossible to measure key properties, such as the internode length, that affect the 598 conduction velocity. Furthermore, the ERP latency is affected by more than the conduction 599 in white matter. The ERP could be affected by processing time (cortical, or sensory), and

600 potentially some properties of the skull and gyrification that could filter the signal. While 601 the inter-individual variance of such properties is still unclear, it is unlikely to be zero. 602 Therefore, one cannot expect to fully explain the data.

603 Despite the aforementioned challenges, it is important to conceptualize the relationship 604 between structural MRI measurements and functional EEG recordings using a biophysical 605 framework. We hope that this work can be used as a first step in bringing these two fields 606 together in an effort to explain common sources for individual differences.

\section{6. Summary}

608 We provide a framework that relates MRI measurements of averaged microstructural 609 features, to a single axon conduction model, in order to be able to predict white matter 610 conduction in vivo. This is a critical step in the process of relating structural MRI 611 measurements to white matter function, in a biologically relevant manner. Such models 612 are highly reductive when we use in vivo measurements that are averaged over a large 613 number of axons. Our results further suggest that using $g$-weighted measurement might 614 not be enough to evaluate meaningful differences in conduction with age. It will be 615 important to take the best available qMRI data (axon diameter estimates included), and a 616 complementary measurement of conduction to show to what extent the models are useful. 617 We hope that as the qMRI models develop further, this sort of study will reveal more about 618 the human brain and behavior in healthy development, aging, and dysfunction. 


\section{Acknowledgments}

621 This work was supported by the Ministry of Science Technology \& Space, Israel (grant 622 no. 3-13395) awarded to S.B. It was also supported by the ISF Grant (no. 0399306) and 623 the NSF/SBE-BSF Grants (NSF no. 1551330 and BSF no. 2015608) awarded to A.A.M., 624 and a seed grant from the Eric Roland Fund for Interdisciplinary Research administered 625 by ELSC, awarded to A.A.M. and S.B.

626 We thank Hiromasa Takemura, Mickey London, Leon Deouel, and Gal Atlan for their 627 helpful comments on this manuscript. 


\section{References}

1. Kanai $R$, Rees $G$. The structural basis of inter-individual differences in human behaviour and cognition. Nat Publ Gr. 2011;12.

2. Zatorre RJ, Fields RD, Johansen-Berg H. Plasticity in gray and white: neuroimaging changes in brain structure during learning. Nat Neurosci. 2012;15(4):528-36.

3. Douglas Fields R. A new mechanism of nervous system plasticity: activitydependent myelination. Nat Publ Gr. 2015;16(12):756.

4. Moeller $\mathrm{K}$, Willmes $\mathrm{K}$, Klein $\mathrm{E}$. A review on functional and structural brain connectivity in numerical cognition. Front Hum Neurosci. Frontiers; 2015;9:227.

5. Nagy Z, Westerberg $\mathrm{H}$, Klingberg $\mathrm{T}$. Maturation of white matter is associated with the development of cognitive functions during childhood. J Cogn Neurosci. 2004;16(7):1227-33.

6. Yeatman JD, Wandell B a, Mezer A a. Lifespan maturation and degeneration of human brain white matter. Nat Commun. 2014;5:4932.

7. Fields RD. White matter matters. Sci Am. 2008;298(3):42-9.

8. Compston A, Coles A. Multiple sclerosis. Lancet. 2008;372(9648):1502-17.

9. Sokol S, Moskowitz A, Towle VL. Age-related changes in the latency of the visual evoked potential: influence of check size 1. Electroencephalogr Clin Neurophysiol. 1981;51:559-62.

10. Reed TE, Vernon PA, Johnson AM. Sex difference in brain nerve conduction velocity in normal humans. Neuropsychologia. 2004;42:1709-14.

11. Polman $\mathrm{CH}$, Reingold SC, Edan G, Filippi M, Hartung H-P, Kappos L, et al. Diagnostic criteria for multiple sclerosis: 2005 revisions to the "McDonald Criteria." Ann Neurol. 2005;58(6):840-6.

12. Assaf $Y$, Pasternak O. Diffusion Tensor Imaging (DTI)-based White Matter Mapping in Brain Research: A Review. J Mol Neurosci. 2008;34(1):51-61.

13. Horsfield M a., Jones DK. Applications of diffusion-weighted and diffusion tensor MRI to white matter diseases - A review. NMR Biomed. 2002;15(7-8):570-7.

14. Gunning-Dixon FM, Brickman AM, Cheng JC, Alexopoulos GS. Aging of cerebral white matter: a review of MRI findings. Int J Geriatr Psychiatry. 2009;24(2):109_ 17.

15. Pumphrey RJ, Young JZ. The rates of conduction of nerve fibres of various diameters in cephalopods. J Exp Biol. 1938;15(4):453-66.

16. Michailov G V, Sereda MW, Brinkmann BG, Fischer TM, Haug B, Birchmeier C, et al. Axonal Neuregulin-1 Regulates Myelin Sheath Thickness. Science (80- ). 
2004;304(5671):700-3.

17. Cullheim S. Relations between cell body size, axon diameter and axon conduction velocity of cat sciatic $\alpha$-motoneurons stained with horseradish peroxidase. Neurosci Lett. 1978;8(1):17-20.

18. Rushton WAH. A theory of the effects of fibre size in medullated nerve. J Physiol. 1951;115(1):101-22.

19. Chomiak T, Hu B. What is the optimal value of the g-ratio for myelinated fibers in the rat CNS? A theoretical approach. PLoS One. 2009;4(11):e7754.

20. Stikov N, Campbell JSW, Stroh T, Lavelée M, Frey S, Novek J, et al. Quantitative analysis of the myelin $\mathrm{g}$-ratio from electron microscopy images of the macaque corpus callosum. Data Br. 2015;4:368-73.

21. Stikov N, Campbell JSW, Stroh T, Lavelée M, Frey S, Novek J, et al. In vivo histology of the myelin g-ratio with magnetic resonance imaging. Neuroimage. 2015;118:397-405.

22. Guy J, Ellis EA, Kelley K, Hope GM. Spectra of G ratio, myelin sheath thickness, and axon and fiber diameter in the guinea pig optic nerve. J Comp Neurol. 1989;287(4):446-54.

23. Arnett $\mathrm{H}$, Mason J, Marino M. TNFa promotes proliferation of oligodendrocyte progenitors and remyelination. Nat Neurosci. 2001;4(11):1116.

24. Pesaresi M, Soon-Shiong R, French L, D.R. Kaplan, Miller FD, Paus T. Axon diameter and axonal transport: In vivo and in vitro effects of androgens. Neuroimage. 2015;115:191-201.

25. Stikov N, Perry ML, Mezer A, Rykhlevskaia E, Wandell B a., Pauly john m., et al. Bound Pool Fractions Complement Diffusion Measures to Describe White Matter Micro and Macrostructure. Neuroimage. 2012;29(6):997-1003.

26. Dean DC, O'Muircheartaigh J, Dirks H, Travers BG, Adluru N, Alexander AL, et al. Mapping an index of the myelin g-ratio in infants using magnetic resonance imaging. Neuroimage. 2016;132:225-37.

27. Berman S, West KL, Does MD, Yeatman JD, Mezer AA. Evaluating g-ratio weighted changes in the corpus callosum as a function of age and sex. Neuroimage. 2018;182:304-13.

28. Duval T, McNab JA, Setsompop K, Witzel T, Schneider T, Huang SY, et al. In vivo mapping of human spinal cord microstructure at $300 \mathrm{mT} / \mathrm{m}$. Neuroimage. 2015;118:494-507.

29. Mohammadi S, Carey D, Dick F, Diedrichsen J, Sereno MI, Reisert M, et al. Whole-Brain In-vivo Measurements of the Axonal G-Ratio in a Group of 37 Healthy Volunteers. 2015;9:441.

30. Caminiti R, Ghaziri H, Galuske R, Hof PR, Innocenti GM. Evolution amplified processing with temporally dispersed slow neuronal connectivity in primates. Proc Natl Acad Sci U S A. 2009;106(46):19551-6. 
31. Westerhausen R, Kreuder F, Woerner W, Huster RJ, Smit CM, Schweiger E, et al. Interhemispheric transfer time and structural properties of the corpus callosum. Neurosci Lett. 2006;409(2):140-5.

32. Horowitz A, Barazany D, Tavor I, Bernstein M, Yovel G, Assaf Y. In vivo correlation between axon diameter and conduction velocity in the human brain. Brain Struct Funct. 2014;220(3):1777-88.

33. Jeeves MA, Moes $P$. Interhemispheric transfer time differences related to aging and gender. Neuropsychologia. Pergamon; 1996;34(7):627-36.

34. Bellis TJ, Wilber LA. Effects of Aging and Gender on Interhemispheric Function. J Speech Lang Hear Res. 2001;44(2):246.

35. Peters A, Moss MB, Sethares $C$. Effects of aging on myelinated nerve fibers in monkey primary visual cortex. J Comp Neurol. 2000;419(3):364-76.

36. Sandell JH, Peters a. Effects of age on nerve fibers in the rhesus monkey optic nerve. J Comp Neurol. 2001;429(4):541-53.

37. Phillips KA, Watson CM, Bearman A, Knippenberg AR, Adams J, Ross C, et al. Age-related changes in myelin of axons of the corpus callosum and cognitive decline in common marmosets. Am J Primatol. 2019 Jan 8;e22949.

38. Hursh JB. Conduction velocity and diameter of nerve fibers. Am J Physiol. 1939;127:131-9.

39. Tasaki I, Ishii K, Ito H. On the Relation between the Conduction-rate, the Fibrediameter and the Internodal Distance of the Medullated Nerve Fibre. Am J Physiol Content. 1955;181(3):639-50.

40. Sanders FK, Whitteridge D. Conduction velocity and myelin thickness in regenerating nerve fibres. J Physiol. John Wiley \& Sons, Ltd (10.1111); 1946 Sep 18;105(2):152-74.

41. Waxman SG, Koscic JD, Stys P k. The Axon: Structure, Function, and Pathophysiology. Oxford University Press, Inc .; 1995.

42. Moore JW, Joyner RW, Brill MH, Waxman SD, Najar-Joa M. Simulations of conduction in uniform myelinated fibers. Relative sensitivity to changes in nodal and internodal parameters. Biophys J. 1978;21(2):147-60.

43. Haltert JA, Clark JW. A Distributed-parameter Model of the Myelinated Nerve Fiber. J theor BioL. 1991;148:345-82.

44. Basser PJ. Cable equation for a myelinated axon derived from its microstructure. Med Biol Eng Comput. 1993;31(1):S87-92.

45. Debanne D, Campanac E, Bialowas A, Carlier E, Alcaraz G. Axon physiology. Physiol Rev. 2011;91(2):555-602.

46. Bakiri Y, Káradóttir R, Cossell L, Attwell D. Morphological and electrical properties of oligodendrocytes in the white matter of the corpus callosum and cerebellum. J Physiol. 2011;589(3):559-73. 
47. Goldman L, Albus JS. Computation of impulse conduction in myelinated fibers; theoretical basis of the velocity-diameter relation. Biophys J. Elsevier; 1968;8(5):596-607.

48. Waxman SG. Determinants of conduction velocity in myelinated nerve fibers. Muscle Nerve. 1980;3(2):141-50.

49. Brill MH, Waxman SG, Moore JW, Joyner RW. Conduction velocity and spike configuration in myelinated fibres: computed dependence on internode distance. $\mathrm{J}$ Neurol Neurosurg Psychiatry. 1977;40(8):769-74.

50. Thomas P., Youl B, Pellisier JF, Huston S, Manson A, Passage E, et al. Correlation of reduction in nerve conduction velocity with varying levels of expression of human peripheral myelin protein 22 (PMP22) in a transgenic mouse model of of demyelinating neuropathy. Electroencephalography and Clinical Neurophysiology. 1997. 12-13 p.

51. Richardson AG, Mclntyre CC, Grill WM. Modelling the effects of electric fields on nerve fibres: Influence of the myelin sheath. Med Biol Eng Comput. SpringerVerlag; 2000 Jul;38(4):438-46.

52. Lorena Arancibia-Cá rcamo I, Ford MC, Cossell L, Ishida K, Tohyama K, Attwell D. Node of Ranvier length as a potential regulator of myelinated axon conduction speed. Elife. 2017;6:e23329.

53. Alexander DC, Dyrby TB, Nilsson M, Zhang H. Imaging brain microstructure with diffusion MRI: practicality and applications. NMR Biomed. 2017;e3841.

54. Drobnjak I, Zhang H, lanuş A, Kaden E, Alexander DC. PGSE, OGSE, and sensitivity to axon diameter in diffusion MRI: Insight from a simulation study. Magn Reson Med. 2016;75(2):688-700.

55. Novikov DS, Fieremans E, Jespersen SN, Kiselev VG. Quantifying brain microstructure with diffusion MRI: Theory and parameter estimation. arXiv Prepr. 2016;arXiv:1612.

56. Assaf $\mathrm{Y}$, Blumenfeld-Katzir T. AxCaliber: a method for measuring axon diameter distribution from diffusion MRI. Magn Reson Med An Off J Int Soc Magn Reson Med. 2008;59(6):1347-54.

57. Kakkar LS, Bennett OF, Siow B, Richardson S, lanuş A, Quick T, et al. Low frequency oscillating gradient spin-echo sequences improve sensitivity to axon diameter: An experimental study in viable nerve tissue. Neuroimage. 2018 Nov;182:314-28.

58. Barakovic M, Girard G, Romascano DPR, Patino Lopez JR, Descoteaux M, Innocenti $G$, et al. Assessing feasibility and reproducibility of a bundle-specific framework on in vivo axon diameter estimates at 300mT/m. 2018.

59. Benjamini D, Komlosh ME, Holtzclaw LA, Nevo U, Basser PJ. White matter microstructure from nonparametric axon diameter distribution mapping. Neuroimage. 2016;135:333-44. 
60. Zhang H, Schneider T, Wheeler-Kingshott CA, Alexander DC. NODDI: Practical in vivo neurite orientation dispersion and density imaging of the human brain. Neuroimage. 2012;61(4):1000-16.

61. Alexander DC, Hubbard PL, Hall MG, Moore EA, Ptito M, Parker GJM, et al. Orientationally invariant indices of axon diameter and density from diffusion MRI. Neuroimage. 2010;52(4):1374-89.

62. Reisert M, Mader I, Umarova R, Maier S, Tebartz van Elst L, Kiselev VG. Fiber density estimation from single q-shell diffusion imaging by tensor divergence. Neuroimage. 2013;77:166-76.

63. Campbell JSW, Leppert IR, Narayanan S, Duval T, Cohen-Adad J, Pike GB, et al. Promise and pitfalls of g-ratio estimation with MRI. 2017;182:80-96.

64. Basser PJ, Jones DK. Diffusion-tensor MRI: theory, experimental design and data analysis - a technical review. NMR Biomed. 2002;15(7-8):456-67.

65. Deoni SCL, Matthews L, Kolind SH. One component? Two components? Three? The effect of including a nonexchanging "free" water component in multicomponent driven equilibrium single pulse observation of $\mathrm{T} 1$ and $\mathrm{T} 2$. Magn Reson Med. 2013;70(1):147-54.

66. Mackay A, Whittall K, Adler J, Li D, Paty D, Graeb D. In vivo visualization of myelin water in brain by magnetic resonance. Magn Reson Med. 1994;31(6):6737.

67. Meyers SM, Kolind SH, MacKay AL. Simultaneous measurement of total water content and myelin water fraction in brain at 3T using a T2 relaxation based method. Magn Reson Imaging. 2017;37:187-94.

68. West KL, Kelm ND, Carson RP, Gochberg DF, Ess KC, Does MD. Myelin volume fraction imaging with MRI. Neuroimage. 2016;182:511-21.

69. Nam Y, Lee J, Hwang D, Kim D-H. Improved estimation of myelin water fraction using complex model fitting. Neuroimage. 2015;116:214-21.

70. Jung W, Lee J, Shin H-G, Nam Y, Zhang H, Oh S-H, et al. Whole brain g-ratio mapping using myelin water imaging (MWI) and neurite orientation dispersion and density imaging (NODDI). Neuroimage. Academic Press; 2017 Sep 27;182:379_ 88.

71. Helms G, Dathe H, Kallenberg K, Dechent P. High-resolution maps of magnetization transfer with inherent correction for RF inhomogeneity and $T 1$ relaxation obtained from 3D FLASH MRI. Magn Reson Med. 2008;60(6):1396407.

72. Sled JG, Pike GB. Quantitative imaging of magnetization transfer exchange and relaxation properties in vivo using MRI. Magn Reson Med. 2001;46(5):923-31.

73. Yarnykh VL. Actual flip-angle imaging in the pulsed steady state: A method for rapid three-dimensional mapping of the transmitted radiofrequency field. Magn Reson Med. 2007;57(1):192-200. 
74. Cercignani M, Giulietti G, Dowell NG, Gabel M, Broad R, Leigh PN, et al. Characterizing axonal myelination within the healthy population: a tract-by-tract mapping of effects of age and gender on the fiber g-ratio. Neurobiol Aging. 2017;49:109-18.

75. Mezer A, Rokem A, Berman S, Hastie T, Wandell BA. Evaluating quantitative proton-density-mapping methods. Hum Brain Mapp. 2016;37(10):3623-35.

76. O'muircheartaigh J, Vavasour I, Ljungberg E, David |, Li KB, Rauscher | Alexander, et al. Quantitative neuroimaging measures of myelin in the healthy brain and in multiple sclerosis. 2019;

77. Ellerbrock I, Mohammadi S. Four in vivo g -ratio-weighted imaging methods: Comparability and repeatability at the group level. Hum Brain Mapp. 2018;39(1):24-41.

78. West KL, Kelm ND, Carson RP, Does MD. A revised model for estimating g-ratio from MRI. Neuroimage. Elsevier B.V.; 2015;

79. Yeatman JD, Dougherty RF, Myall NJ, Wandell B a., Feldman HM. Tract Profiles of White Matter Properties: Automating Fiber-Tract Quantification. PLoS One. 2012;7(11).

80. Tournier J-D, Calamante F, Connelly A. MRtrix: Diffusion tractography in crossing fiber regions. Int J Imaging Syst Technol. 2012;22(1):53-66.

81. Smith SM, Jenkinson M, Johansen-Berg H, Rueckert D, Nichols TE, Mackay CE, et al. Tract-based spatial statistics: Voxelwise analysis of multi-subject diffusion data. Neuroimage. 2006;31(4):1487-505.

82. De Santis S, Drakesmith M, Bells S, Assaf Y, Jones DK. Why diffusion tensor MRI does well only some of the time: Variance and covariance of white matter tissue microstructure attributes in the living human brain. Neuroimage. 2014;89:35-44.

83. Hermes D, Nguyen M, Winawer J. Neuronal synchrony and the relation between the blood-oxygen-level dependent response and the local field potential. Kohn A, editor. PLOS Biol. 2017;15(7):e2001461.

84. Eyal G, Verhoog MB, Testa-Silva G, Deitcher Y, Lodder JC, Benavides-Piccione $\mathrm{R}$, et al. Unique membrane properties and enhanced signal processing in human neocortical neurons. Elife. 2016;5:e16553.

85. Young KM, Psachoulia K, Tripathi RB, Dunn S-J, Cossell L, Attwell D, et al. Oligodendrocyte Dynamics in the Healthy Adult CNS: Evidence for Myelin Remodeling. Neuron. 2013;77(5):873-85.

86. Friede RL. The Significance of Internode Length for Saltatory Conduction: Looking Back at the Age of 90. J Neuropathol Exp Neurol. 2017;76(4):258-9.

87. Etxeberria A, Hokanson KC, Dao DQ, Mayoral SR, Mei F, Redmond SA, et al. Dynamic Modulation of Myelination in Response to Visual Stimuli Alters Optic Nerve Conduction Velocity. J Neurosci. 2016;36(26):6937-48.

88. Friede RL, Bischhausen R. How are sheath dimensions affected by axon caliber 
and internode length? Brain Res. 1982;235(2):335-50.

89. Aboitiz F, Scheibel a B, Fisher RS, Zaidel E. Fiber composition of the human corpus callosum. Brain Res. 1992;598(1-2):143-53.

90. Ibrahim ' M, Butt AM, Berry M. Relationship between myelin sheath diameter and internodal length in axons of the anterior medullary velum of the adult rat. $J$ Neurol Sci. 1995;133(1):119-27.

91. West KL, Kelm ND, Carson RP, Does MD. A revised model for estimating g-ratio from MRI. Neuroimage. 2016;125:1155-8.

92. Moes PE, Brown WS, Minnema MT. Individual differences in interhemispheric transfer time (IHTT) as measured by event related potentials. Neuropsychologia. 2007;45(11):2626-30.

93. Whitford TJ, Kubicki M, Ghorashi S, Schneiderman JS, Hawley KJ, McCarley RW, et al. Predicting inter-hemispheric transfer time from the diffusion properties of the corpus callosum in healthy individuals and schizophrenia patients: A combined ERP and DTI study. Neuroimage. 2011;54(3):2318-29.

94. Patston LLM, Kirk IJ, Rolfe MHS, Corballis MC, Tippett LJ. The unusual symmetry of musicians: Musicians have equilateral interhemispheric transfer for visual information. Neuropsychologia. 2007;45(9):2059-65.

95. Terada K, Umeoka S, Usui N, Baba K, Usui K, Fujitani S, et al. Uneven interhemispheric connections between left and right primary sensori-motor areas. Hum Brain Mapp. 2012;33(1):14-26.

96. Terada K, Usui N, Umeoka S, Baba K, Mihara T, Matsuda K, et al. Interhemispheric Connection of Motor Areas in Humans. J Clin Neurophysiol. 2008;25(6):351-6.

97. Marzi CA, Bisiacchi P, Nicoletti R. Is interhemispheric transfer of visuomotor information asymmetric? Evidence from a meta-analysis. Neuropsychologia. $1991 ; 29(12): 1163-77$.

98. Mezer A, Yeatman JD, Stikov N, Kay KN, Cho N-J, Dougherty RF, et al. Quantifying the local tissue volume and composition in individual brains with magnetic resonance imaging. Nat Med. 2013;19(12):1667-72.

99. Barral JK, Gudmundson E, Stikov N, Etezadi-Amoli M, Stoica P, Nishimura DG. A robust methodology for in vivo T1 mapping. Magn Reson Med. 2010;64(4):105767.

100. Mezer A, Rokem A, Berman S, Hastie T, Wandell BA. Evaluating quantitative proton-density-mapping methods. Hum Brain Mapp. 2016;37(10):3623-35.

101. Jenkinson M, Beckmann CF, Behrens TEJ, Woolrich MW, Smith SM. FSL. Neuroimage. 2012;62(2):782-90.

102. Andersson JLR, Skare S, Ashburner J. How to correct susceptibility distortions in spin-echo echo-planar images: application to diffusion tensor imaging.

Neuroimage. 2003;20(2):870-88. 
103. Andersson JLR, Sotiropoulos SN. An integrated approach to correction for offresonance effects and subject movement in diffusion MR imaging. Neuroimage. 2016;125:1063-78.

104. Jenkinson M, Bannister P, Brady M, Smith S. Improved optimization for the robust and accurate linear registration and motion correction of brain images. Neuroimage. 2002;17(2):825-41.

105. Jenkinson $\mathrm{M}$, Smith S. A global optimisation method for robust affine registration of brain images. Med Image Anal. 2001;5(2):143-56.

106. Daducci A, Canales-Rodríguez EJ, Zhang H, Dyrby TB, Alexander DC, Thiran J$P$. Accelerated Microstructure Imaging via Convex Optimization (AMICO) from diffusion MRI data. Neuroimage. 2015;105:32-44.

107. Ikeda M, Oka Y. The relationship between nerve conduction velocity and fiber morphology during peripheral nerve regeneration. Brain Behav. John Wiley \& Sons, Ltd; 2012 Jul 1;2(4):382-90.

108. Agmon-Snir H, Segev I. Signal delay and input synchronization in passive dendritic structures. J Neurophysiol. 1993;70(5):2066-85.

109. Rall W. Core conductor theory and cable properties of neurons. Handb Physiol Nerv Syst I. 2011;39-97.

110. Budde MD, Annese J. Quantification of anisotropy and fiber orientation in human brain histological sections. Front Integr Neurosci. Frontiers; 2013 Feb 1;7:3.

111. Drakesmith $M$, Jones DK. Mapping axon conduction delays in vivo from microstructural MRI. bioRxiv. Cold Spring Harbor Laboratory; 2018 Dec $21 ; 503763$.

112. Innocenti GM, CarlÃ M, Dyrby TB. Chapter15. The Diameters of Cortical Axons and Their Relevance to Neural Computing. Axons Brain Archit. 2015;317-36.

113. Perge J a, Niven J, Mugnaini E, Balasubramanian V, Sterling P. Why Do Axons Differ in Caliber? 2012;32(2):626-38.

114. Perge J a, Koch K, Miller R, Sterling P, Balasubramanian V. How the optic nerve allocates space, energy capacity, and information. J Neurosci. 2009;29(24):791728.

115. Xu J, Li H, Harkins KD, Jiang X, Xie J, Kang H, et al. Mapping mean axon diameter and axonal volume fraction by MRI using temporal diffusion spectroscopy. Neuroimage. 2014;103:10-9.

116. Fieremans E, Burcaw LM, Lee H-H, Lemberskiy G, Veraart J, Novikov DS. In vivo observation and biophysical interpretation of time-dependent diffusion in human white matter. Neuroimage. 2016;129:414-27.

117. Forkel SJ, Mahmood S, Vergani F. The white matter of the human cerebrum: Part I The occipital lobe by Heinrich Sachs. Cortex. Elsevier; 2015 Jan 1;62:182-202.

118. Bürgel U, Schormann T, Schleicher A, Zilles K. Mapping of Histologically 
Identified Long Fiber Tracts in Human Cerebral Hemispheres to the MRI Volume of a Reference Brain: Position and Spatial Variability of the Optic Radiation. Neuroimage. Academic Press; 1999 Nov 1;10(5):489-99.

119. Berman S, West KL, Does MD, Yeatman JD, Mezer AA. Evaluating g-ratio weighted changes in the corpus callosum as a function of age and sex. Neuroimage. 2018;182:304-13.

120. Luck S. Ten simple rules for designing ERP experiments. Event-related potentials A methods Handb. 2005;17-32.

121. Brown WS, Jeeves MA. Bilateral visual field processing and evoked potential interhemispheric transmission time. Neuropsychologia. 1993;31(12):1267-81.

122. Fendrich R, Hutsler J, Gazzaniga M. Visual and tactile interhemispheric transfer compared with the method of Poffenberger. Exp Brain Res. 2004;158(1):67-74. 


\section{Supplementary figures}

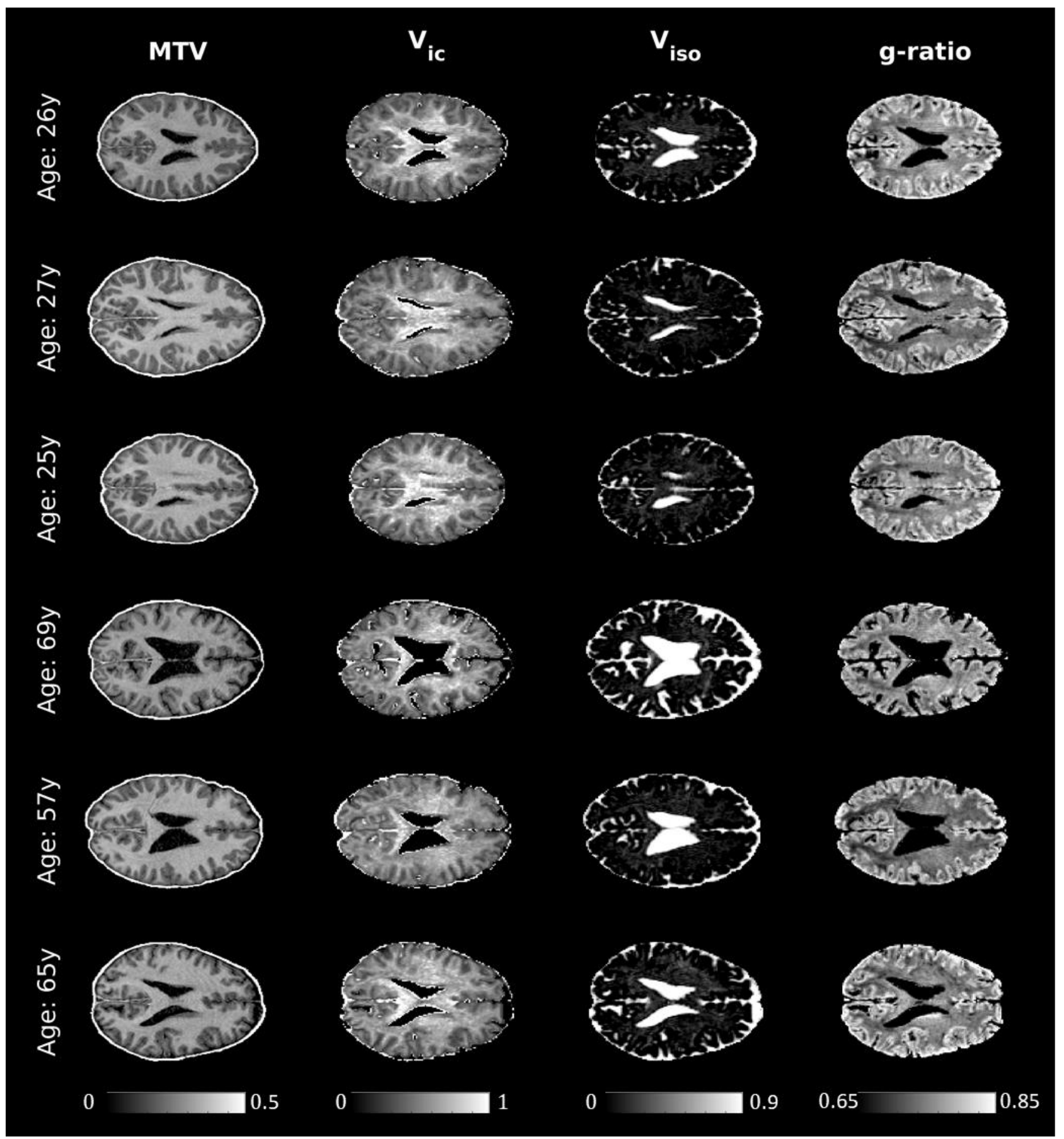

Supplementary Figure 1: whole brain g-ratio maps. Examples of the qMRI maps used in this study, for 3 younger and 3 older subjects. The maps presented here are the MTV (the non-water fraction), the intracellular and isotropic volume fraction $\left(V_{i c}\right.$ and $V_{i s o}$, respectively), calculated as parameters in the NODDI model. The g-ratio is calculated from $M T V, V_{i c}$ and $V_{i s o}$. 
bioRxiv preprint doi: https://doi.org/10.1101/479881; this version posted January 27,2019 . The copyright holder for this preprint (which was not certified by peer review) is the author/funder, who has granted bioRxiv a license to display the preprint in perpetuity. It is made available under aCC-BY-NC-ND 4.0 International license.
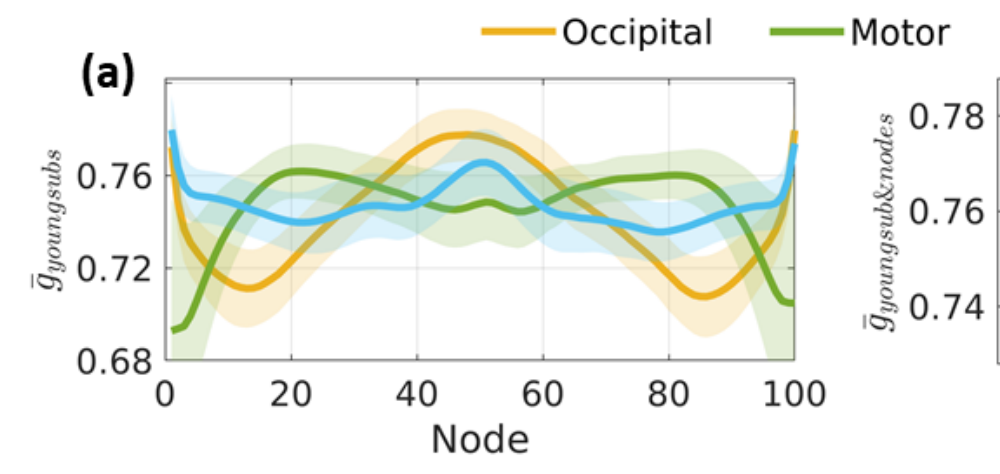

- Ant-Frontal
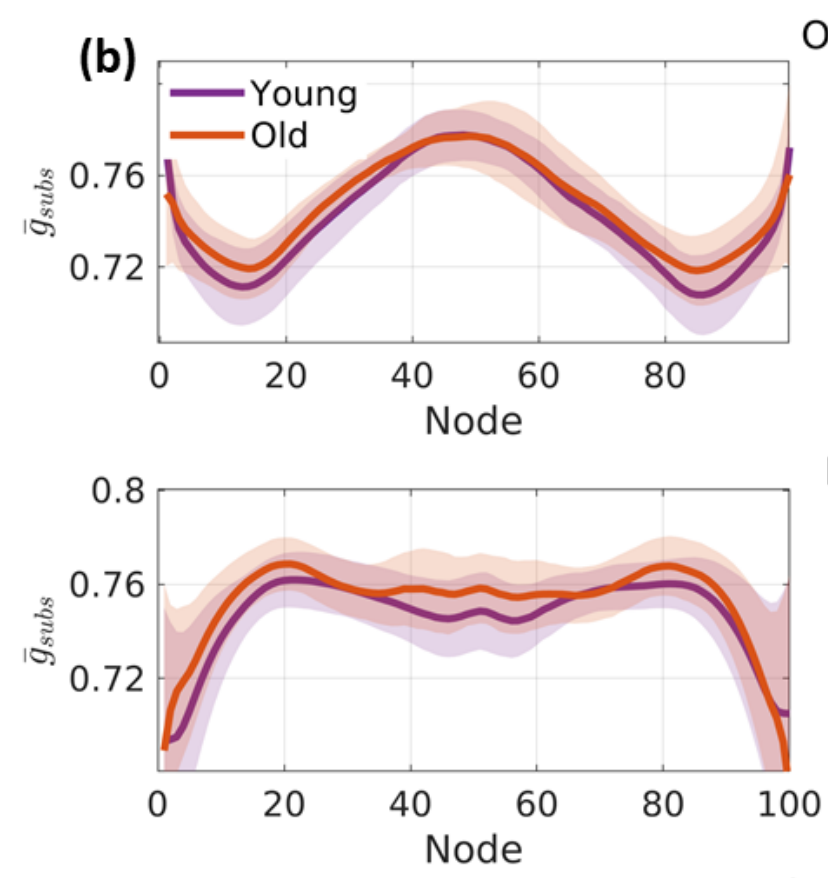

Occipital

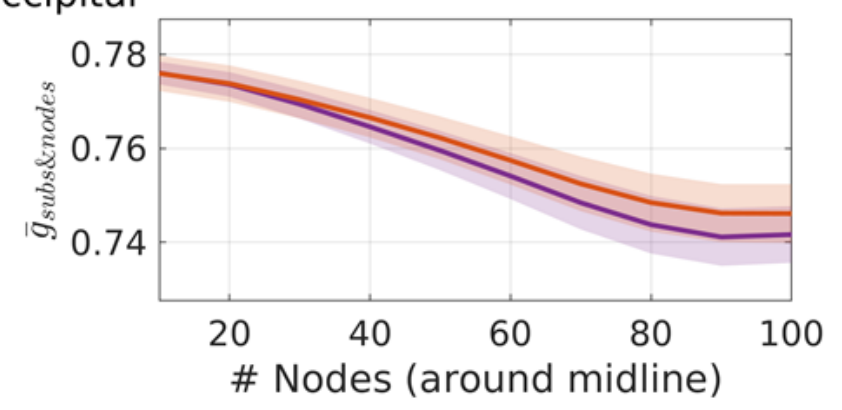

Motor

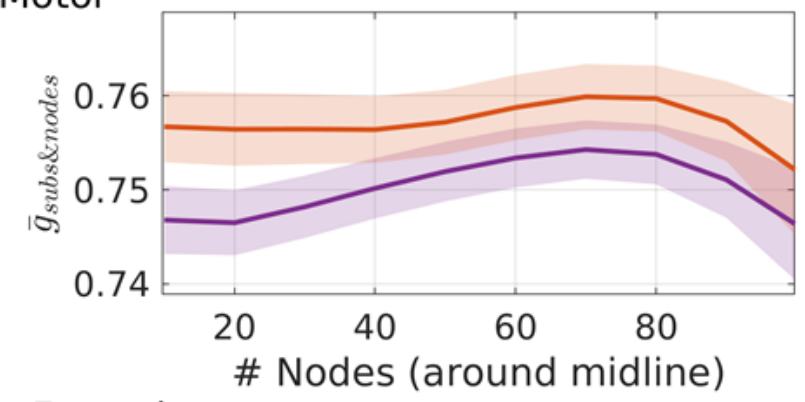

Ant-Frontal
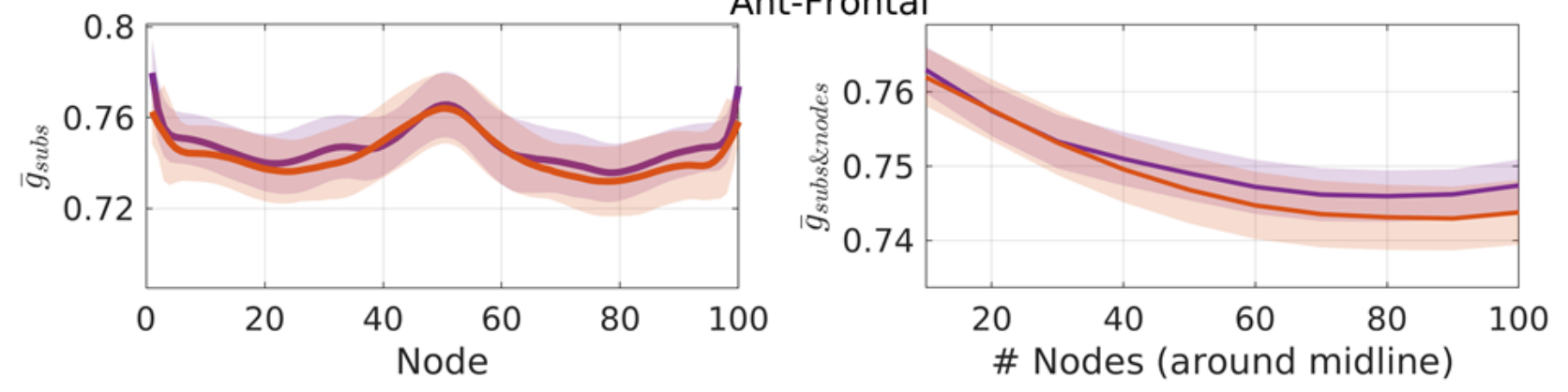

Supplementary Figure 2: Variance along the fiber tract. (a) The left plot shows the average g across younger subjects at each node along the fiber tract (100 nodes, from left to right). The shaded area represents the STD at each node. The three tracts show different relationships with respect to the node location. To characterize the $\mathrm{g}$ of a tract, we average over several nodes. The right plot is the average as a function of the number of nodes taken around the midline (node 50), from 10 nodes to the entire tract (shaded area represents STD). The relationship between the callosal tracts changes as a function of the number of nodes taken to calculate the mean. (b) The plots in (a) are shown separately for the different fiber tracts, comparing the average $g$ of younger (purple) and older (orange) subjects. While there are slight variations in the age-group differences as a function of node-number, the overall relationship remains the same. 


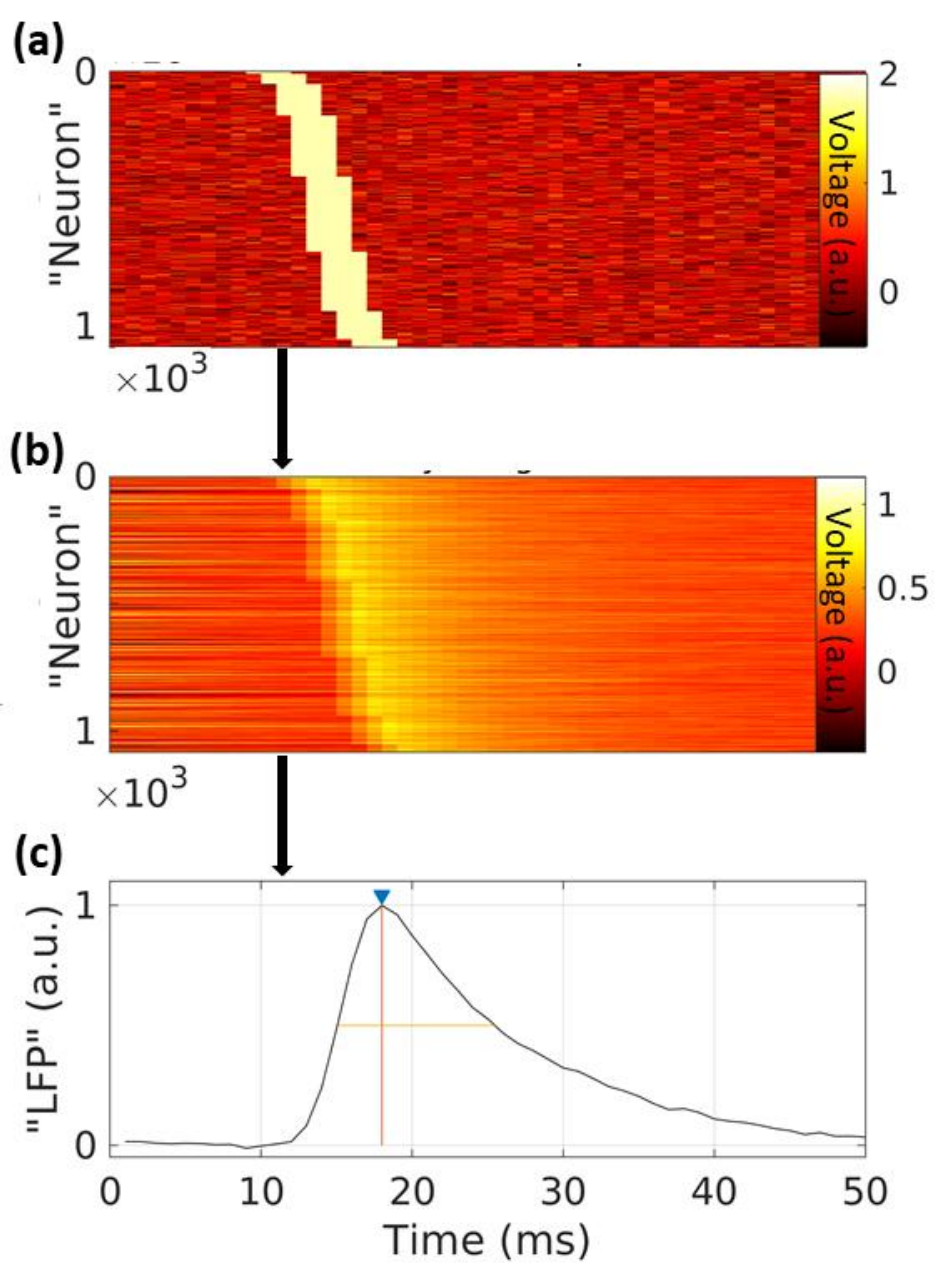

Supplementary Figure 3: LFP simulation. The distribution of latencies, calculated from the streamlines' g-ratio and length, is used as input for the simulation of leaky-integrating neurons. (a) The input is modeled as Gaussian noise, with a delta function of the corresponding latency of the streamline. (b) The input is then leaky-integrated over time. (c) The LFP is the sum over the integrated time series. The signal is normalized to have a peak of 1 [a.u]. Finally, we calculated the peak time and the full width half maximum of the ERP. 